\title{
Self-assembling micrites based on the Platonic solids
}

\author{
Johndale C. Solem \\ Theoretical Division, Los Alamos National Laboratory, Los Alamos, NM 87545, USA
}

Received 29 May 2000; received in revised form 29 May 2001

Communicated by T.C. Henderson

\begin{abstract}
Micrites capable of assembling into arbitrary shapes in three dimensions, with the constraint of a single species, might be most effectively fabricated in the shape of Platonic solids (regular polyhedra), to take advantage of maximum symmetry and to ensure that each polygon face of one micrite will perfectly coincide with the face of another micrite, thereby maximizing adhesion and minimizing stray fields. Only two of the regular polyhedra can be assembled to fill all space: the cube and the dodecahedron. Therefore, only the cube and dodecahedron are capable of constructing arbitrary shapes including the highest possible strength realized by a voidless solid. The dihedral angle between the adjoining faces of two cubes, joined by coinciding squares on a face of each cube, is $180^{\circ}$. Hence, it is difficult for an electric field, generated by charges on the perpendicular faces, to rotate the two cubes in such a way as to join at another pair of faces. The dodecahedron, however, has a corresponding dihedral angle of about $127^{\circ}$ and a pair of dodecahedra can be easily caused to roll from one contact face to another. Therefore, the dodecahedron is the only space-filling Platonic solid that is also capable of easily-generated face-field-driven motion.

I demonstrate that the micrites must be maintained in a fluid environment in order to provide sufficient lubrication for their polygonal binding surfaces to azimuthally rotate into coincidence. I calculate the speed of convergence in a lubricant environment and show that the distant micrites assemble only very slowly (for a practical range of parameters) unless there is some agitation of the fluid. I show how various solids can be constructed by convergence. I demonstrate how a primitive motor can be built from two dodecahedron-shaped micrites, and I calculate the maximum speed of the motor and the maximum power the motor can generate. I explain a surprisingly simple algorithm for a curious self-propelling flagellum constructed from a chain of dodecahedron-shaped micrites. I calculate the flagellum's maximum swimming speed and power consumption. Published by Elsevier Science B.V.
\end{abstract}

Keywords: Self-assembly; Microrobot

\section{Introduction}

In 1991, this researcher investigated the possibility of constructing microrobots using photon lithographic techniques, particularly, such microrobots that could be fabricated close to the limit of X-ray lithographic technology. These previously considered microrobots had a multitude of moving parts, and special atten-

E-mail address: jxcs@lanl.gov (J.C. Solem). tion was paid to their locomotion [1,2]. This paper is concerned with mechanical self-organization of microrobots with no moving parts at all. Micrites ${ }^{1}$ considered in this paper must interact with each other

\footnotetext{
${ }^{1}$ ARPA has used the term micrite to designate a microrobot with these properties. The term micrite already has a petrologic definition as a semiopaque micro-crystalline limestone matrix that consists of chemically precipitated calcite mud. While this may cause some confusion, I will use the term coined by ARPA.
} 
through electric fields produced by charges on their faces. Because it is most convenient and most durable for the micrites to bind at faces that are all the same size and all the same shape (regular polygons), micrites of most general applicability must be regular solids, otherwise known as Platonic solids. The five Platonic solids in ordinary space are: (1) the tetrahedron, (2) the cube, (3) the octahedron, (4) the dodecahedron, and (5) the icosahedron. It is convenient to characterize these solids by the Schläfli [3] symbol, $\{p, q\}$, where $p$ is the number of edges on each face and $q$ is the number of faces joined by each vertex, whereby we have: tetrahedron $\{3,3\}$, cube $\{4,3\}$, octahedron $\{3,4\}$, dodecahedron $\{5,3\}$, and icosahedron $\{3,5\}$ [4]. The surface area of any Platonic solid is

$$
S=\frac{p q l^{2}}{4-(p-2)(q-2)} \cot \left(\frac{\pi}{p}\right),
$$

where $l$ is the length of an edge. Similarly, the volume is given by

$$
\begin{aligned}
V_{\mathrm{ol}}= & \frac{1}{6} \frac{p q l^{3}}{4-(p-2)(q-2)} \cot ^{2}\left(\frac{\pi}{p}\right) \cos \left(\frac{\pi}{q}\right) \\
& \cdot \csc \left(\frac{\pi}{2}\left(\frac{10-p-q}{p+q+2}\right)\right) .
\end{aligned}
$$

The dihedral angle between the planes of any two adjoining faces of the same Platonic solid is

$\phi=2 \arcsin \left[\cos \left(\frac{\pi}{q}\right) \csc \left(\frac{\pi}{p}\right)\right]$.

When two regular polyhedra are joined by coinciding polygons, the dihedral angle between the faces of the two at the joining plane is

$\varphi=2 \pi-4 \arcsin \left[\cos \left(\frac{\pi}{q}\right) \csc \left(\frac{\pi}{p}\right)\right]$.

The radii of the circum-sphere $\left({ }_{0} R\right)$, which passes through all the vertices, the mid-sphere $\left({ }_{1} R\right)$, which touches all the edges, and the in-sphere $\left({ }_{2} R\right)$, which touches all the faces, are related by

${ }_{0} R^{2}=4 l^{2}+{ }_{1} R^{2}=4 l^{2} \csc ^{2}\left(\frac{\pi}{p}\right)+{ }_{2} R^{2}$

and

${ }_{1} R^{2}=4 l^{2} \cot ^{2}\left(\frac{\pi}{p}\right)+{ }_{2} R^{2}$,

where $p$ is again the first Schläfli symbol $\{p, q\}$.

\section{Alignment of the contact surface}

Contact between faces can be promoted by either an electric or a magnetic field. From practical considerations on the micron level, this discussion will be specialized to electric fields. Then the surfaces will consist of an electrical conductor coated with a thin layer of insulator to suppress currents when the micrites come into contact. The surfaces by which the Platonic micrites adhere to each other are three regular polygons: triangle, square, and pentagon.

Will the micrites adhere in such a manner that the faces coincide? If so the pattern will always be regular and the maximum binding energy (or tensile strength) will be achieved.

\subsection{Transverse alignment}

For each of the polygons, the coefficient of friction that will allow the surfaces to align with each other is dependent on the azimuthal alignment in a straightforward, but complicated way. The essential physics is preserved and greatly simplified if we approximate the polygon surfaces with a circle of the same area. The areal overlap between two circles of radius $R$, whose centers are displaced by a distance $b$ is given by

$$
A=2\left[R^{2} \arccos \left(\frac{b}{2 R}\right)-\frac{1}{2} b \sqrt{R^{2}-\frac{b^{2}}{4}}\right],
$$

where $0 \leqslant b \leqslant 2 R$. The electrical energy in a parallel plate capacitor is

$W=\frac{1}{2} \epsilon \frac{A}{d} V^{2}$,

where $A$ is the overlap area, $d$ is the distance between the two parallel plates, $\epsilon$ is the permittivity of the dielectric between the plates, and $V$ is the voltage applied to the plates. So the force sliding the circular plates into alignment is

$F_{\|}=\frac{\mathrm{d} W}{\mathrm{~d} b}=-\frac{\epsilon R V^{2}}{2 d} \sqrt{4-\frac{b^{2}}{R^{2}}}$,

where the $\|$ indicates the force is parallel to the plates and the minus sign indicates a restoring force applied to bring the disks back to $b=0$. The force pulling the two plates together in the direction normal to their 
surfaces is

$$
\begin{aligned}
F_{\perp} & =\frac{\epsilon A V^{2}}{2 d^{2}} \\
& =\frac{\epsilon V^{2}}{d^{2}}\left[R^{2} \arccos \left(\frac{b}{2 R}\right)-\frac{1}{2} b \sqrt{R^{2}-\frac{b^{2}}{4}}\right] .
\end{aligned}
$$

Neglecting the detailed dynamics, the circles will not be able to slide into alignment unless

$F_{\|}>\mu F_{\perp}$,

where $\mu$ is the coefficient of sliding friction. Combining Eqs. (9)-(11), the disks move into alignment if

$\mu<-\frac{R d \sqrt{4-b^{2} / R^{2}}}{2 R^{2} \arccos (b / 2 R)+b \sqrt{R^{2}-b^{2} / 4}}$.

If the disks are nearly aligned, i.e. $b \ll R$, then

$\mu \simeq \frac{2 d}{\pi R}$

so for a $100 \mu \mathrm{m}$ radius surface, with a $1 / 2 \mu \mathrm{m}$ dielectric coating, the surface must have a sliding friction coefficient $\mu<0.003$. Such a coefficient cannot be achieved without lubrication.

\subsection{Rotational alignment}

We now consider the case where the polygons attach coaxially, but with random azimuthal angles. The maximal energy attachment will occur if the overlapping surface area is

$A_{\max }=n r^{2} \tan \frac{\pi}{n}$,

where $n$ is the number of sides and $r$ the radius of the inscribed circle of the polygon. When one of the polygons is rotated $\pi / n$, the minimal energy attachment will occur and the overlapping surface area is

$A_{\min }=2 n r^{2} \tan \frac{\pi}{2 n}$.

So the energy changes from minimum to maximum when one of the polygons is rotated $\Delta \theta=\pi / n$. Combining Eqs. (14), (15) and (8), the difference between the maximum and minimum energy is

$\Delta W=\frac{\epsilon n r^{2} V^{2}}{2 d}\left(\tan \frac{\pi}{n}-2 \tan \frac{\pi}{2 n}\right)$.
We know that $\mathrm{d} W / \mathrm{d} \theta=0$ at both $\theta=0$ and $\theta=\pi / n$, but the functions $W(\theta)$ and $\mathrm{d} W / \mathrm{d} \theta$ are complicated. It is much easier to write the average electrostatic torque

$\bar{\tau}=\frac{\Delta W}{\Delta \theta}=\frac{\epsilon n^{2} r^{2} V^{2}}{2 \pi d}\left(\tan \frac{\pi}{n}-2 \tan \frac{\pi}{2 n}\right)$.

From Eq. (9), the force between the plates is

$F_{\perp} \simeq \frac{\pi r^{2} \epsilon V^{2}}{2 d^{2}}$

and the frictional torque resisting rotation is

$\bar{\tau} \simeq \mu \frac{\pi r^{3} \epsilon V^{2}}{4 d^{2}}$

where $\mu$ is the coefficient of friction. Equating Eqs. (17) and (19), we have the approximate alignment criterion

$\mu<\frac{2 n^{2} d}{\pi^{2} r}\left(\tan \frac{\pi}{n}-2 \tan \frac{\pi}{2 n}\right)$,

or in numerical form,

$\mu<\frac{d}{r} \begin{cases}1.053, & \text { triangle }(n=3) \\ 0.556, & \text { square }(n=4) \\ 0.389, & \text { pentagon }(n=5) .\end{cases}$

As for the transverse alignment, azimuthal alignment for reasonable values of $d$ and $r$ cannot be ensured without lubrication.

Thus, the micrites must be immersed in a fluid lubricant of some sort. It would also be desirable to give the micrites neutral buoyancy within the fluid, so gravity can be neglected and the electrostatic and viscous forces alone are in play.

\section{Lubrication}

Reynolds number is used to describe the relative importance of inertial forces and viscous forces. It is always given for a specific geometric shape; and it scales with linear dimension $\xi$ such that

$R_{\mathrm{e}} \sim \frac{\rho v \xi}{\eta}$

where $\rho$ is the fluid density, $v$ is the velocity through the fluid, and $\eta$ is the fluid viscosity. For exceedingly small objects, inertial forces are swamped by viscous forces. 
The viscosity of SAE 30 motor oil is about 4.0 dyn s cm$~_{-2}$ at $40{ }^{\circ} \mathrm{C}$, its density about $0.9 \mathrm{~g} \mathrm{~cm}^{-3}$, its dielectric constant is about six times that of vacuum, and its resistivity is about $2 \times 10^{16} \Omega \mathrm{cm}^{-3}$. A micrite about $1 \mathrm{~mm}$ in linear dimension, moving about $0.1 \mathrm{~mm} \mathrm{~s}^{-1}$ would have a Reynolds number of about $2 \times 10^{-5}$. Its motion would be strongly dominated by viscous forces, and inertial and gravitational forces can be neglected.

For a Newtonian fluid between two planar plates parallel to the $x-y$ plane of a Cartesian coordinate system, the shear stress $\sigma$ and pressure $p$ are related by

$\frac{\partial p}{\partial x}=\frac{\partial \sigma}{\partial z}$,

and the viscosity $\eta$ is defined such that

$\sigma=\eta \frac{\partial u}{\partial z}$,

where $u$ is the fluid velocity. Substituting Eq. (24) into Eq. (23), we obtain

$\frac{\mathrm{d} p}{\mathrm{~d} x}=\eta \frac{\partial^{2} u}{\partial z^{2}}$,

assuming the plates are large compared to their separation and $\eta$ is everywhere constant [5]. Integrating Eq. (25) twice and applying the boundary condition $u=0$ at both surfaces, we obtain

$u=\frac{1}{2 \eta} \frac{\mathrm{d} p}{\mathrm{~d} x} z(z-d)$

where $d$ is again the distance between the plates.

\subsection{Approximate equations of motion for converging micrites}

In understanding the kinetics of micrites being drawn together by electric fields, it is necessary to understand the motions involved in: (1) terminal convergence, when the faces are nearly touching; (2) translation, when the faces are nearly touching but not yet aligned; and (3) distant convergence, when the micrites are being drawn together by their dipole-dipole interaction, each moving in the field gradient of the other.

\subsubsection{Terminal convergence}

As before we will approximate the faces of the Platonic solids with circular disks, to take advantage of the simplification offered by cylindrical symmetry. From Eq. (26), the total fluid flow rate between two parallel coaxial disks is

$Q=2 \pi r \int_{0}^{d} u \mathrm{~d} z=-2 \pi r \frac{h^{3}}{12 \eta} \frac{\mathrm{d} p}{\mathrm{~d} r}$.

Taking $p=0$ at the outer radius $R$, the viscous force holding the disks apart is

$$
\begin{aligned}
F_{\perp} & =2 \pi \int_{0}^{R} p R \mathrm{~d} R=-\pi \int_{0}^{R} R^{2} \frac{\mathrm{d} p}{\mathrm{~d} R} \mathrm{~d} R \\
& =\frac{6 \eta Q}{d^{3}} \int_{0}^{R} R \mathrm{~d} R=\frac{3 \eta Q R^{2}}{d^{3}},
\end{aligned}
$$

where $r$ is the distance from the center of the disks. The volume between the disks is $2 \pi R^{2} d$, so

$Q=2 \pi R^{2} \dot{d}$.

The electrostatic force pulling the parallel coaxial disks together is

$F_{\perp}=\frac{\epsilon A V^{2}}{2 d^{2}}=\frac{\epsilon \pi R^{2} V^{2}}{2 d^{2}}$.

Combining Eqs. (28)-(30) and integrating, the distance between the plates will shrink according to

$d=d_{0} \exp \left(-\frac{\epsilon V^{2}}{12 \eta R^{2}} t\right)$.

In this approximation, the plates will never make contact, and, if the micrites make contact, the insulator on the micrite surface will stop the closure. The approximation is good only if the insulator is thin. The time for two micrites to converge to a distance $d$ is obtained from Eq. (31) to be

$t=\frac{12 \eta R^{2}}{\epsilon V^{2}} \ln \frac{d_{0}}{d}$,

nominal size of the micrites is $1 \mathrm{~mm}$. From Eqs. (5) and (6), the radius of the dodecahedral in-sphere is

${ }_{2} R=\frac{(1+\sqrt{5})^{5 / 2}}{8(20)^{1 / 4}}=1.11 l$.

So, if the micrite is a dodecahedron with diametric face distance of $2_{2} R=1 \mathrm{~mm}$, the length of its edge 
would be $2 l=0.449 \mathrm{~mm}$, and, from Eq. (1), the surface area of one pentagonal face is

$$
\begin{aligned}
A & =\frac{5}{4} l^{2} \cot \left(\frac{\pi}{5}\right) \simeq 1.72 l^{2} \simeq 0.347 \mathrm{~mm}^{2} \\
& =3.47 \times 10^{-7} \mathrm{~m}^{2} .
\end{aligned}
$$

For clarity, the diametric face distance is the perpendicular distance between the two parallel pentagons on opposite sides of the dodecahedron. In Figs. 3(a) and 3(b), the parallel pentagons would be numbered: 1 and $2 ; 3$ and $4 ; 5$ and $6 ; \ldots ; 11$ and 12 . A circle with area $3.47 \mathrm{~mm}^{2}$ has radius $R=3.32 \times 10^{-4} \mathrm{~m}$. The permittivity of pure water is $\epsilon=7.18 \times 10^{-10}$ in practical (mks) units at $20^{\circ} \mathrm{C}$, and its viscosity is about $\eta=0.0101$ dyn s cm ${ }^{-2}=1.01 \times 10^{-3} \mathrm{~J} \mathrm{~s} \mathrm{~m}^{-3}$. If we take the micrites to be initially $1 \mathrm{~mm}$ apart each with a face potential of $10 \mathrm{~V}$ (a potential of $20 \mathrm{~V}$ between the two converging faces) and say they are for most intents and purposes in contact when they are $1 \mu \mathrm{m}$ apart, Eq. (32) gives a time to contact is about $32.1 \mathrm{~ms}$. For comparison, the permittivity of motor oil is about six times vacuum or $\epsilon=5.31 \times 10^{-11}$ (mks) and its viscosity is about $\eta=4 \times 10^{-1} \mathrm{~J} \mathrm{~s} \mathrm{~m}^{-3}$. With a face potential of $100 \mathrm{~V}$ the time to contact is about $1.72 \mathrm{~s}$.

\subsubsection{Translation}

The frictional force between two lubricated surfaces is

$F_{\|} \simeq \frac{\eta v_{\|} A}{d}$,

where $A$ is again the contact surface area and $v_{\|}$is the velocity with which they are being drawn across each other. Remarkably, when this is equated to the electrostatic force pulling the plates into alignment (Eq. (9)), the distance between the plates drops out, and the velocity of alignment is

$$
\begin{aligned}
v_{\|}= & -\frac{\epsilon R V^{2}}{4 \eta} \sqrt{4-\frac{b^{2}}{R^{2}}} \\
& \times\left[R^{2} \arccos \left(\frac{b}{2 R}\right)-\frac{1}{2} b \sqrt{R^{2}-\frac{b^{2}}{4}}\right]^{-1},
\end{aligned}
$$

which is singular at $b=2 R$, but presents no problem for subsequent integrations. Assuming they start at a center-to-center spacing of $b$, the time required for them to align will be

$$
\begin{aligned}
t= & \int_{b}^{0} \frac{\mathrm{d} b}{v_{\|}} \\
= & -\frac{b^{2} \eta}{V^{2} \epsilon}-\frac{8 \eta R}{\epsilon V^{2}} \int_{b}^{0} \frac{R^{2}}{\sqrt{R^{2}-b^{2}}} \\
& \times \arccos \left(\frac{b}{2 R}\right) \mathrm{d} b .
\end{aligned}
$$

The integral on the far right of Eq. (37) is not easily evaluated, but we can use the approximation

$$
\frac{R^{2}}{\sqrt{R^{2}-b^{2}}} \arccos \left(\frac{b}{2 R}\right) \simeq 0.773-0.142 \frac{b}{R}
$$

which is within a few percent of the function value on the interval $0 \leqslant b \leqslant 2 R$. So from Eqs. (37) and (38), we obtain the approximation

$t \simeq \frac{1.57 \eta}{\epsilon V^{2}}\left(3.94 b R-b^{2}\right)$.

The time for osculating disks to become concentric $(b=2 R)$ is then

$t \simeq \frac{6.10 \eta R^{2}}{\epsilon V^{2}}$.

Using the values for SAE 30 weight oil, a micrite face $100 \mu \mathrm{m}$ in radius $(R=100 \mu \mathrm{m})$, and a face potential of $100 \mathrm{~V}$, we find the time to attain concentricity is about $115 \mu \mathrm{s}$, quite fast on the human time scale.

\subsubsection{Distant convergence}

At distances large compared to the micrite diameter, the micrite appears as an electric multipole. If only diametric plates are charged, the micrite appears as a dipole $p=q D$, where $D$ is the face-to-face distance and $\pm q$ is the charge on each plate and $q$ is in statcoulombs (esu or dyn ${ }^{1 / 2} \mathrm{~cm}$ ), where $1 \mathrm{C}=$ $2.996 \times 10^{9}$ statcoulombs. Two similar micrites interact by torquing each other and by attraction in their mutual electric fields assuming they quickly re-orient to minimize potential. The torque on a micrite in and electric field is

$\vec{L}=\vec{p} \times \vec{E}$.

At a distance $\left|\vec{r}-\vec{r}_{0}\right| \gg D$, the electric field from either of the micrites is

$\vec{E}(\vec{r})=\frac{3 \vec{n}(\vec{p} \cdot \vec{n})-\vec{p}}{\epsilon\left|\vec{r}-\vec{r}_{0}\right|^{3}}$, 
where $\vec{r}_{0}$ is the location of the dipole and $\vec{n}$ is the unit vector directed form $\vec{r}_{0}$ to $\vec{r}$. The initial torque on micrite $\mathrm{A}$ is

$\vec{L}_{\mathrm{A}}=\vec{p}_{\mathrm{A}} \times \frac{3 \vec{n}\left(\vec{p}_{\mathrm{B}} \cdot \vec{n}\right)-\vec{p}_{\mathrm{B}}}{\epsilon\left|\vec{r}_{\mathrm{A}}-\vec{r}_{\mathrm{B}}\right|^{3}}$,

where $\vec{p}_{\mathrm{A}}$ and $\vec{p}_{\mathrm{B}}$ are the dipole moments of micrites $\mathrm{A}$ and $\mathrm{B}$, respectively. If, e.g. micrite $\mathrm{B}$ has its dipole parallel to the line connecting the micrites and micrite A has its dipole perpendicular to the line connecting the micrites, Eq. (43) becomes

$L_{\mathrm{A}}=\frac{4 p^{2}}{\epsilon r^{3}}$,

where $r=\left|\vec{r}_{\mathrm{A}}-\vec{r}_{\mathrm{B}}\right|$ and $p=\left|\vec{p}_{\mathrm{A}}\right|=\left|\vec{p}_{\mathrm{B}}\right|$. The torque is also small.

The interaction energy between two micrites with dipole moments is

$W_{\mathrm{AB}}=\frac{\vec{p}_{\mathrm{A}} \cdot \vec{p}_{\mathrm{B}}-3\left(\vec{n} \cdot \vec{p}_{\mathrm{A}}\right)\left(\vec{n} \cdot \vec{p}_{\mathrm{B}}\right)}{\epsilon\left|\vec{r}_{\mathrm{A}}-\vec{r}_{\mathrm{B}}\right|^{3}}$.

If the micrites have their dipoles aligned along the line connecting them, Eq. (45) becomes

$W_{\mathrm{AB}}=\frac{4 p^{2}}{\epsilon r^{3}}$.

The force felt by each dipole attracting it toward the other is

$F_{\mathrm{AB}}=-\frac{12 p^{2}}{\epsilon r^{4}}$.

The drag force on a sphere of radius $R$ is $6 \pi \eta R v$, where $v$ is its velocity in the viscous fluid. Equating this drag force to the attractive force given in Eq. (47) and integrating we find the time it will take the micrites to converge to where they are in each others near field is given by

$t \simeq \frac{\pi \eta \epsilon R}{10 p^{2}} r^{5}$,

assuming that the viscous forces are large compared to the inertial forces. At this point it is easy to observe that the micrites will not influence each other much in the far field. The time for them to converge goes as the fifth power of the distance between them!

To put this into numerical context, consider again that our micrite can be regarded as a parallel-plate capacitor

$C=\frac{q}{V}=\frac{\epsilon_{\mathrm{m}} A}{4 \pi d}$, where $\epsilon_{\mathrm{m}}$ is the dielectric constant of the micrite material. Combining Eq. (49) with Eq. (48) and using $p=q d$, we obtain

$t \simeq \frac{8 \pi^{3} \epsilon \eta R}{5 A^{2} \epsilon_{\mathrm{m}}^{2} V^{2}} r^{5}$.

Again consider our dodecahedron micrite with diametric face distance of $1 \mathrm{~mm}$, corresponding to an edge length of $l=0.449 \times 10^{-3} \mathrm{~m}$. The area of one pentagonal face is $A=3.47 \mathrm{~mm}^{2}=3.47 \times 10^{-6} \mathrm{~m}^{2}$. The volume of a dodecahedron is obtained from Eq. (2) as

$$
\begin{aligned}
V_{\mathrm{ol}} & =\frac{5}{4} \cot ^{2}\left(\frac{\pi}{5}\right) \csc \left(\frac{\pi}{10}\right) l^{3} \simeq 7.66 l^{3} \\
& \simeq 4.27 \times 10^{-10} \mathrm{~m}^{3} .
\end{aligned}
$$

The dodecahedron is approximately a sphere so Stokes' law will be a good approximation. A sphere of the same volume as the dodecahedron $V_{\mathrm{ol}} \simeq 4.27 \times$ $10^{-10} \mathrm{~m}^{3}$ will have a radius of $R=4.67 \times 10^{-4} \mathrm{~m}$. Take a pair of these micrites immersed in water $\left(\epsilon=7.18 \times 10^{-10}\right.$ (mks), $\left.\eta=1.01 \times 10^{-3} \mathrm{~J} \mathrm{~s} \mathrm{~m}^{-3}\right)$ across which the potential is $10 \mathrm{~V}$. Also take $\epsilon_{\mathrm{m}}=\epsilon$. The time to converge to the near field from a separation $r=1 \mathrm{~cm}$ is $2.71 \times 10^{3} \mathrm{~s}$ or about $45 \mathrm{~min}$. If the initial separation were $10 \mathrm{~cm}$, convergence would require $2.71 \times 10^{8} \mathrm{~s}$ or about 8.5 years!

Clearly, for the micrites to self-assemble, there must be some agitation of the fluid in which they are immersed. This agitation has already been found necessary when millimeter-scale objects were selfassembled into two-dimensional shapes using wetability of the interfaces to provide binding [6].

\section{Space-filling}

For most general utility, the micrites must be capable of filling all space. The reason is strength: a solid member of some construction will have greatest tensile strength if the constituent micrites can bind to each other without voids. If a member has voids, its tensile strength will be diminished from the maximum electric-interface strength by a factor of

$\frac{A_{\mathrm{t}}-A_{\mathrm{v}}}{A_{\mathrm{t}}}$

where $A_{\mathrm{t}}$ is the total cross-sectional area of the member at the binding surfaces and $A_{\mathrm{v}}$ is the void 
cross-sectional area of voids at the binding surfaces. For example, a member built from solid-packed cubes would have twice the tensile strength of one with only every other cube filled in. In general, the tensile strength will be anisotropic.

\subsection{The space-filling Platonic solids}

The cube is the basic unit of Cartesian coordinates in three dimensions. There is only one noncubic tesselation of three-space with regular polyhedra: the dodecahedron.

\subsubsection{Sketch of proof for mathematicians}

Find two regular polyhedra, the sum of whose dihedral angles is $2 \pi / n$, where $n$ is an integer. These can only be the octahedron and the tetrahedron, and each vertex of the tesselation joins six octahedra and eight tetrahedra. The vertices are all alike. The symmetry group is the same as for the cuboctahedron, which has 12 vertices. Therefore, each vertex joins 12 lines, twice as many as the cubic tesselation.

\subsubsection{Sketch of proof for physicists}

Consider a face-centered cubic lattice (FCC) lattice. Draw lines between the lattice points and construct perpendicular planes bisecting these lines. There are 12 lines from each point, so the planes will form regular dodecahedra, which fill all space.

Because the cube $\{4,3\}$ and the dodecahedron $\{5,3\}$ are the only space filling regular solids, and thereby capable of composing structural elements of maximum tensile strength, we restrict our attention to these two polyhedra.

\section{The cube $\{4,3\}$}

From Eq. (1), the surface area of a cube is $6 l^{2}$ and, from Eq. (2), its volume is $l^{3}$. If we are restricted to electric dipoles between the faces of a cube, the number of such dipoles is (6 taken 2 at a time) times 2 polarities, which is

$2 \frac{6 !}{2 !(6-2) !}=30$.

An indefinite number of dipole directions and strengths is possible if we can fractionally charge the faces.

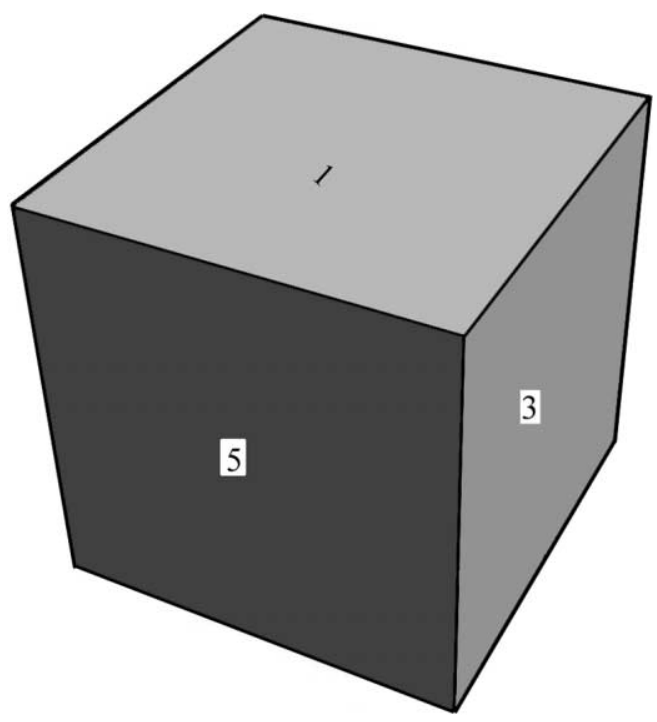

Fig. 1. Numbering scheme used to distinguish among the six faces of the cube. Visible are only the odd numbered faces. The face diametric to each of these faces is found by adding 1, e.g. the face diametric to 1 is 2 , the face diametric to 5 is 6 .

From Eq. (3), the dihedal angle between the faces of a cube is $90^{\circ}$. When two cubes are joined by coinciding squares, the dihedral angle between the faces of the two at the joining plane is $180^{\circ}$, as given by Eq. (4). Once two cubes are electrostatically bound at a common face, they cannot easily be made to rotate to a new face by applying charges to those faces.

Fig. 1 shows the numbering scheme used here to distinguish among the six faces of the cube. Visible in the figure are only the odd numbered faces. The face diametric to each of these faces is found by adding 1, e.g. the face diametric to 1 is 2 , the face diametric to 5 is 6 .

\subsection{Making a filament}

A filament may be constructed by applying plus and minus charges to sequential faces. For example, a ' + ' charge could be applied to all faces 1 , while a '-' charge is applied to all faces 2 . Because there is some physical flexing at the faces where the cubes bind, the filament will not necessarily be a straight line, and several filaments may form at once. Fig. 2 is a simulation of the assembly of a filament from a collection of five cubic micrites. The frame of reference in Fig. 2 is such that the location and orientation of the bottom cube is constant. 


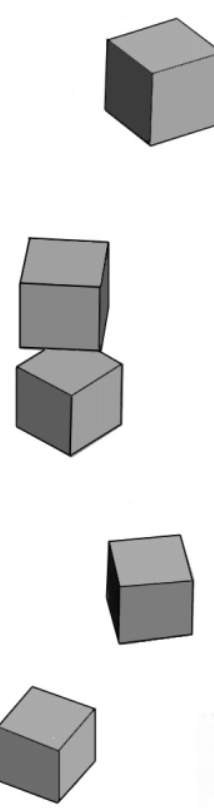

(a)

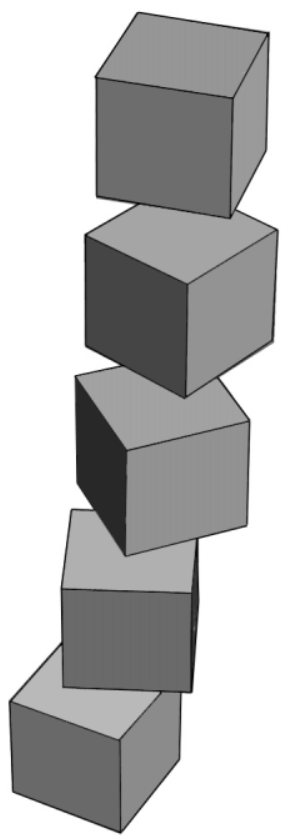

(d)
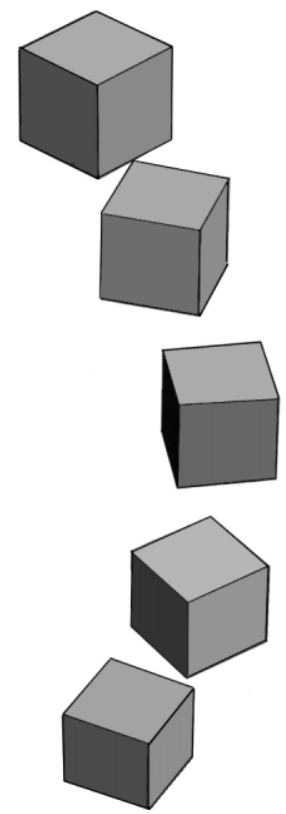

(b)

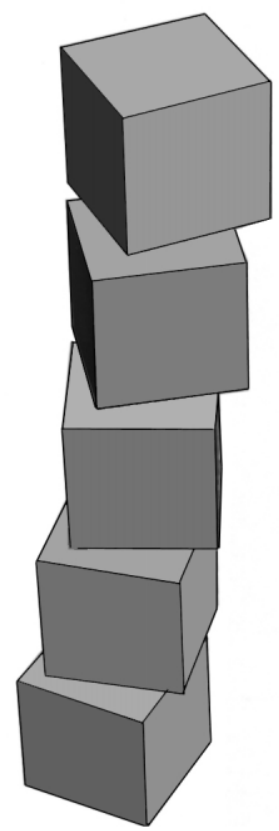

(e)

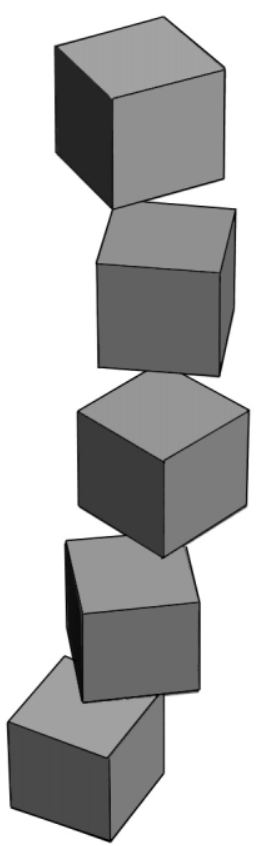

(c)

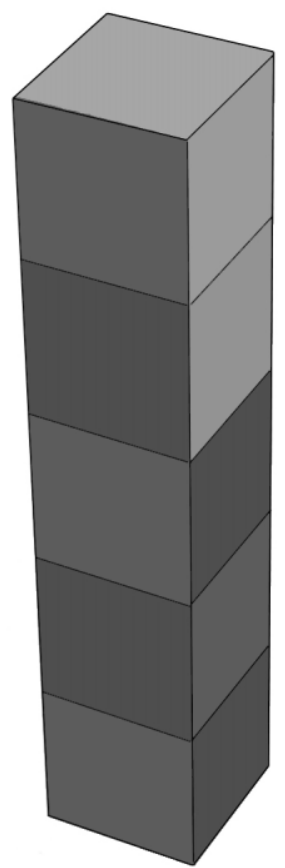

(f)

Fig. 2. A simulation of the assembly of a filament from a collection of five cubic micrites. Frame of reference is such that the location and orientation of the bottom cube is constant. Arbitrary-unit time sequence is (a), (b), (c), ( d), (e), (f). 
If we command all the polyhedra to apply a potential between faces 1 and 2, that is, move charge from face 1 to face 2, the micrites will line up as shown in Fig. 2(a), which shows the micrites in a column. Because of lubrication, they will eventually line up with coinciding faces as shown in Fig. 2(f). Depending on how high the potential for stochastic bending of the filament, it may form a loop as well.

\section{The dodeahedron $\{5,3\}$}

From Eq. (1), the surface area of a dodecahedron is $\sim 20.6 l^{2}$ and, from Eq. (2), its volume is $\sim 7.66 l^{3}$. From Eq. (14), the dihedal angle between the faces of a dodecahedron is $116.57^{\circ}$. If we are restricted to electric dipoles between the faces of a cube, the number of such dipoles is ( 12 taken 2 at a time) times 2 polarities, which is

$2 \frac{12 !}{2 !(12-2) !}=132$.

Once two dodecahedra are electrostatically bound at a common face, they can be made to rotate to a new face by applying opposite charges to those faces. From Eq. (4), the dihedral angle between those faces is $126.87^{\circ}$, whereas it is $180^{\circ}$ for cubes.

Fig. 3 shows the numbering scheme used here to distinguish among the 12 faces of the dodecahedron. As in Fig. 1, only the odd numbered faces are visible.

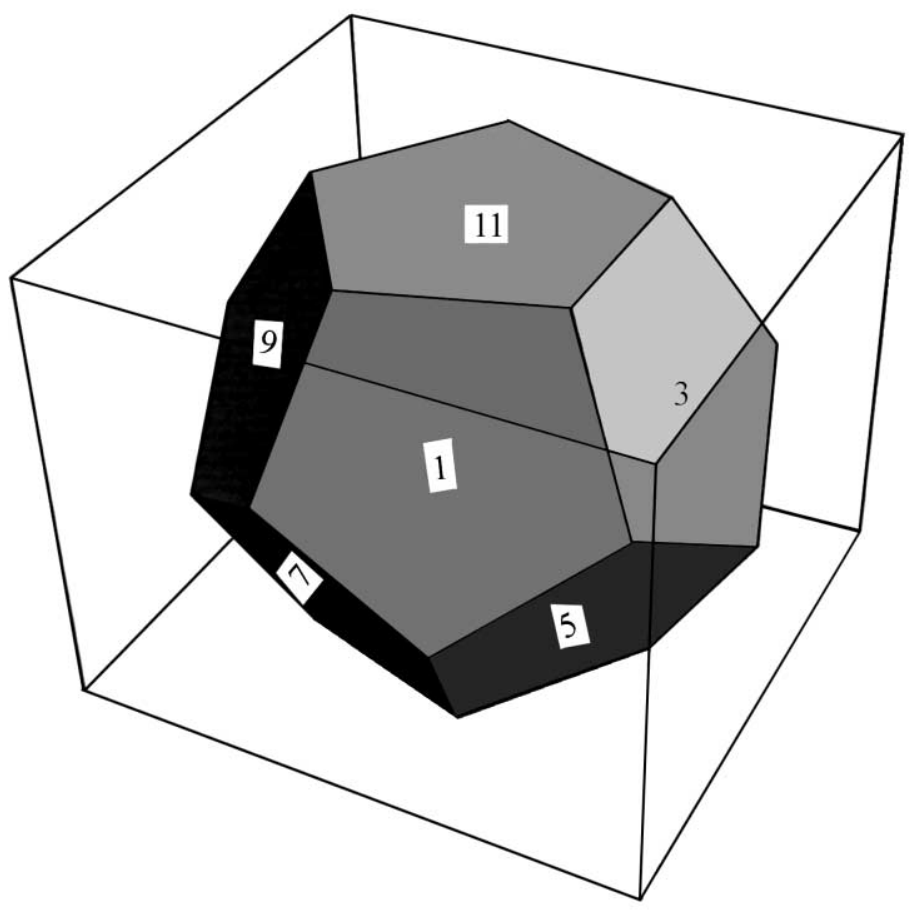

(a)

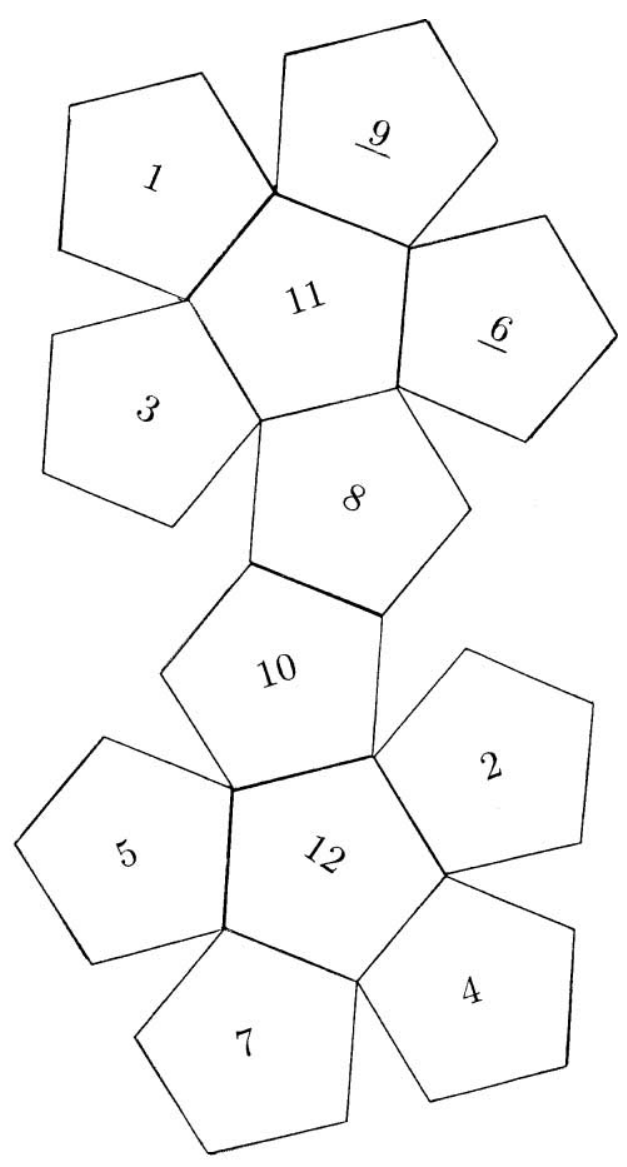

(b)

Fig. 3. (a) Numbering scheme used to distinguish among the 12 faces of the dodecahedron. As in Fig. 1, only the odd numbered faces are visible. The face diametric to each of these faces is again found by adding 1. (b) Faces of the dodecahedron unfolded onto a plane. 
The face diametric to each of these faces is again found by adding 1 , e.g. the face diametric to 5 is 6 , the face diametric to 11 is 12 . Fig. 3(b) shows the faces of the dodecahedron unfolded onto a plane.

\subsection{Making a filament}

If we command all the dodecahedra to apply a potential between faces 1 and 2, that is, move charge from face 1 to face 2, the micrites will line up as shown in Fig. 4, which shows the micrites in a column. Because azimuthal energy will be minimized, the lubricated convergence to an aligned filament will involve some rotation of the dodecahedra, as illustrated for the cube in Fig. 2. Depending on how high the potential for stochastic bending of the filament, it could also form a loop.

\subsection{Making planar and solid structures}

Fig. 5 is a simulation of the convergence of a dodecahedron on a pair of bound dodecahedra, forming a triangle of dodecahedra, which will be the basic building block of an undulating plane. The plane is a face-centered cubic structure, Fig. 6 shows the centers of the dodecahedra with a box delineating the surfaces of the planar structure. To a crystallographer's eye, the centers of the dodecahedra form a face-centered cubic lattice, which is to be expected from their symmetry. Fig. 7 shows the centers of dodecahedra formed into a rough-surfaced sphere with maximum diameter of 151 dodecahedra.

\subsection{Making a primitive motor}

Fig. 8(a) shows two micrites bound together with a ' + ' charge on face 11 of the lower micrite (specified micrite B) and a '-' charge on face 12 of the upper (specified micrite A). (Recall the definitions of the face numbers on the dodecahedron as given in Figs. 3(a) and (b).) The charges on are then turned off and a ' + ' is applied to face 3 of micrite A, while a ' - ' is applied to face 5 of micrite B. Figs. 8(a)-8(e) show the sequence of events as the micrites roll to their new positions. In each case the balancing charge is applied to the face diametric to the contacting face. A repetition of the same pattern causes the two micrites to revolve around each other constituting a primitive

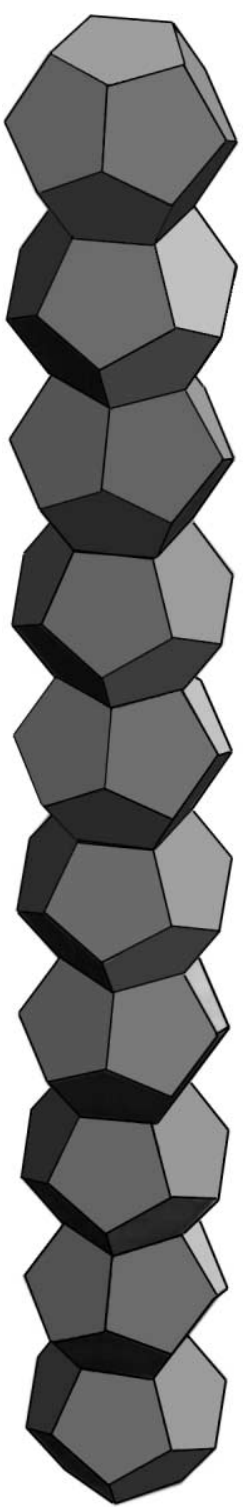

Fig. 4. If we command all the dodecahedra to apply a potential between faces 1 and 2, they will align in a filament. Because of lubrication, dodecahedral micrites line up with coinciding faces.

motor. The exact sequence of events is summarized in Table 1, where $t$ is dimensionless time.

Several symmetries are immediately apparent. The commands being given to both micrite $\mathrm{A}$ and micrite $\mathrm{B}$ are exactly the same. A motor in the opposite direction is obtained by the mirror image or initially 

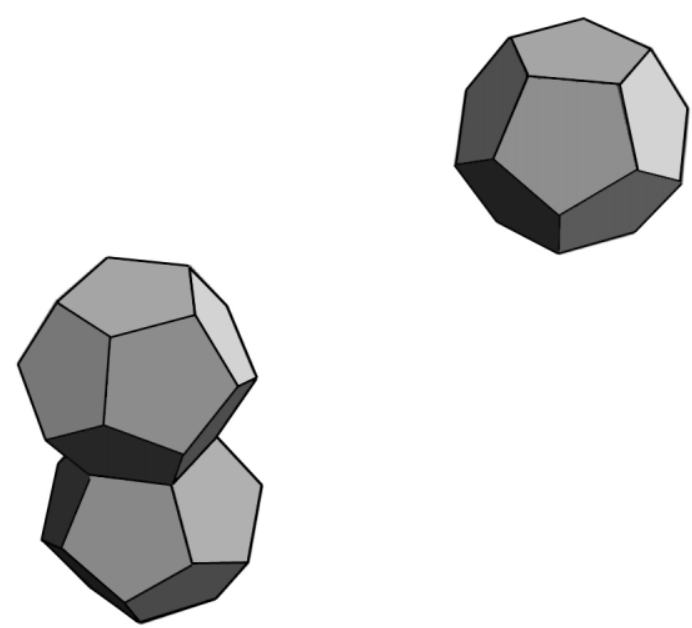

(a)

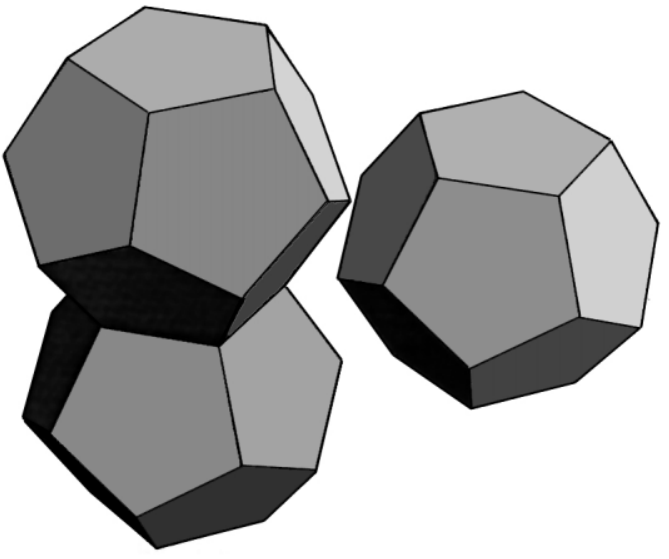

(c)
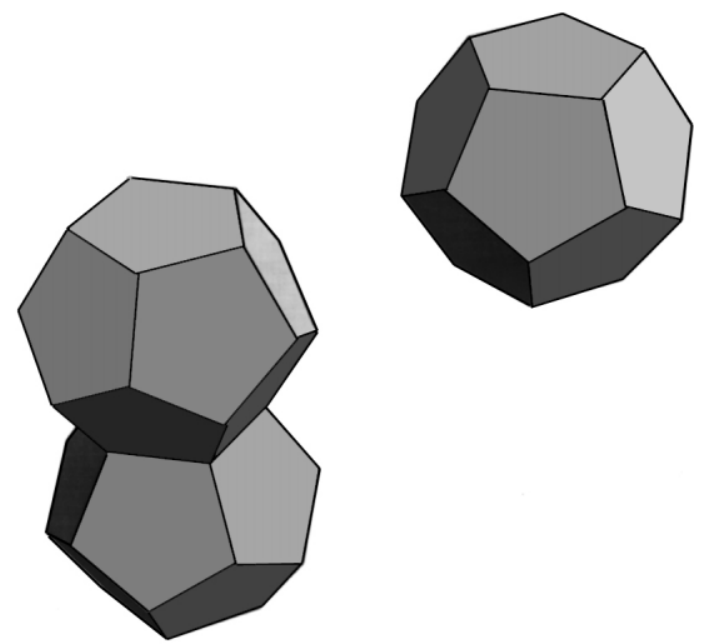

(b)

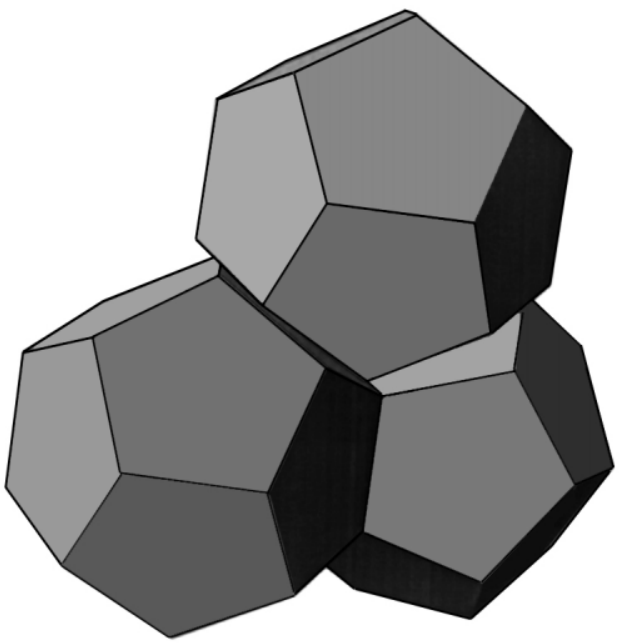

(d)

Fig. 5. Simulation of the convergence of a dodecahedron on a pair of bound dodecahedra, forming a triangle of dodecahedra, which will be the basic building block of an undulating plane. Arbitrary-unit time sequence is (a), (b), (c), (d).

interchanging micrite A and micrite $\mathrm{B}$. The same will be obtained by reversing the charges. The faces with ' + ' and ' - ' are given as a function of time by the formula

$F_{ \pm}=2\lfloor t \bmod 5\rfloor+\frac{7}{2} \pm \frac{1}{2}$

The floor ' $\mathrm{J}$ ' retains integer values for the face numbers despite fractional (real) values of time.
Table 1 describes a microprocessor program to make the two micrites revolve around each other. It could be used as a switching program just as described, as providing the time interval of the charge rotation is long enough to allow closure of the faces and alignment of the pentagons. With more elaborate electronics, it could include a feedback loop to ensure that contact and alignment are attained between faces before charging the next face in the rotation. 


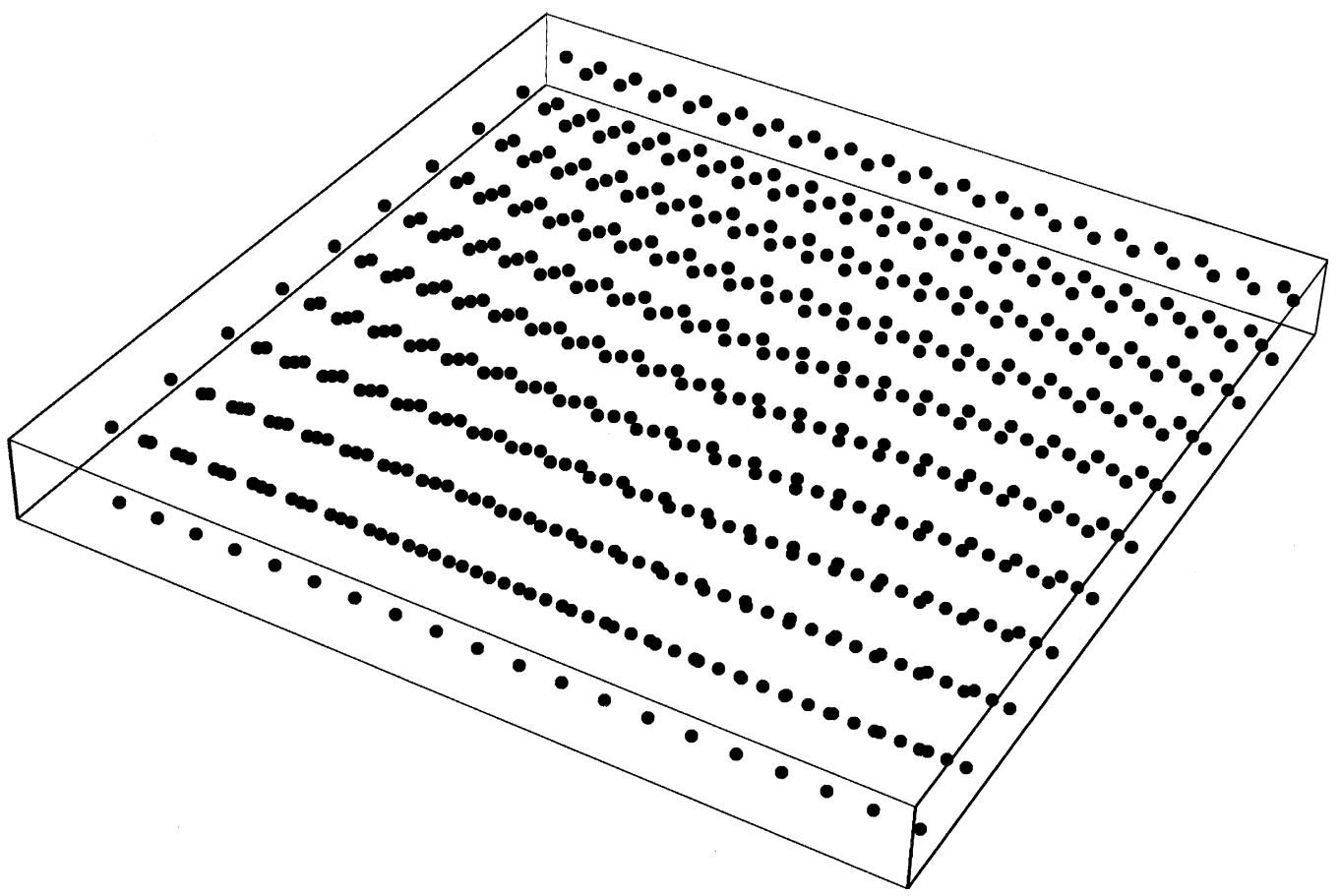

Fig. 6. Centers of the dodecahedra with a box delineating the surfaces of the planar structure.

Capacitance between the faces is minimum when they are opened to their maximum dihedral angle and maximum when the faces are closed and aligned. Assuming that the micrites are emersed in a dielectric fluid, charge could be moved from the face that is going to close to any or all of the 11 remaining faces. For motions involving more than two micrites, such as the flagellum described later, there always remain several faces to which charge can be moved without affecting

Table 1

Time sequence of charges on faces of two dodecahedral micrites for primitive motor

\begin{tabular}{rrrrr}
\hline$t$ & $\mathrm{~A}_{-}$ & $\mathrm{A}_{+}$ & $\mathrm{B}_{-}$ & $\mathrm{B}_{+}$ \\
\hline 0 & 3 & 4 & 3 & 4 \\
1 & 5 & 6 & 5 & 6 \\
2 & 7 & 8 & 7 & 8 \\
3 & 9 & 10 & 9 & 10 \\
4 & 11 & 12 & 11 & 12 \\
5 & 3 & 4 & 3 & 4 \\
6 & 5 & 6 & 5 & 6 \\
7 & 7 & 8 & 7 & 8 \\
$\vdots$ & $\vdots$ & $\vdots$ & $\vdots$ & $\vdots$ \\
\hline
\end{tabular}

the revolution of the micrites. Detecting contact can then be accomplished by either of two methods:

1. Constant charge: The microprocessor and attendant electronics maintain a constant charge on the closing surface while monitoring the voltage required to maintain that charge. The voltage will decrease as the faces close, will reach a minimum when the faces contact, and remain at that minimum. The microprocessor has then received a positive signal that the faces are closed and then rotates voltage to the next face of the sequence given in Table 1 .

2. Constant voltage: The microprocessor and attendant electronics maintain a constant voltage on the closing surface (with respect to the other faces of the micrite) while monitoring the current flowing to the face. The greatest current will occur while the dihedral angle is in the process of closing, and the current will go to zero when the faces are closed. The current will also go to zero when motion has stopped for any reason, thereby detecting interference with the motion.

At a higher level of sophistication, variable charges and voltages might also be used to improve efficiency 


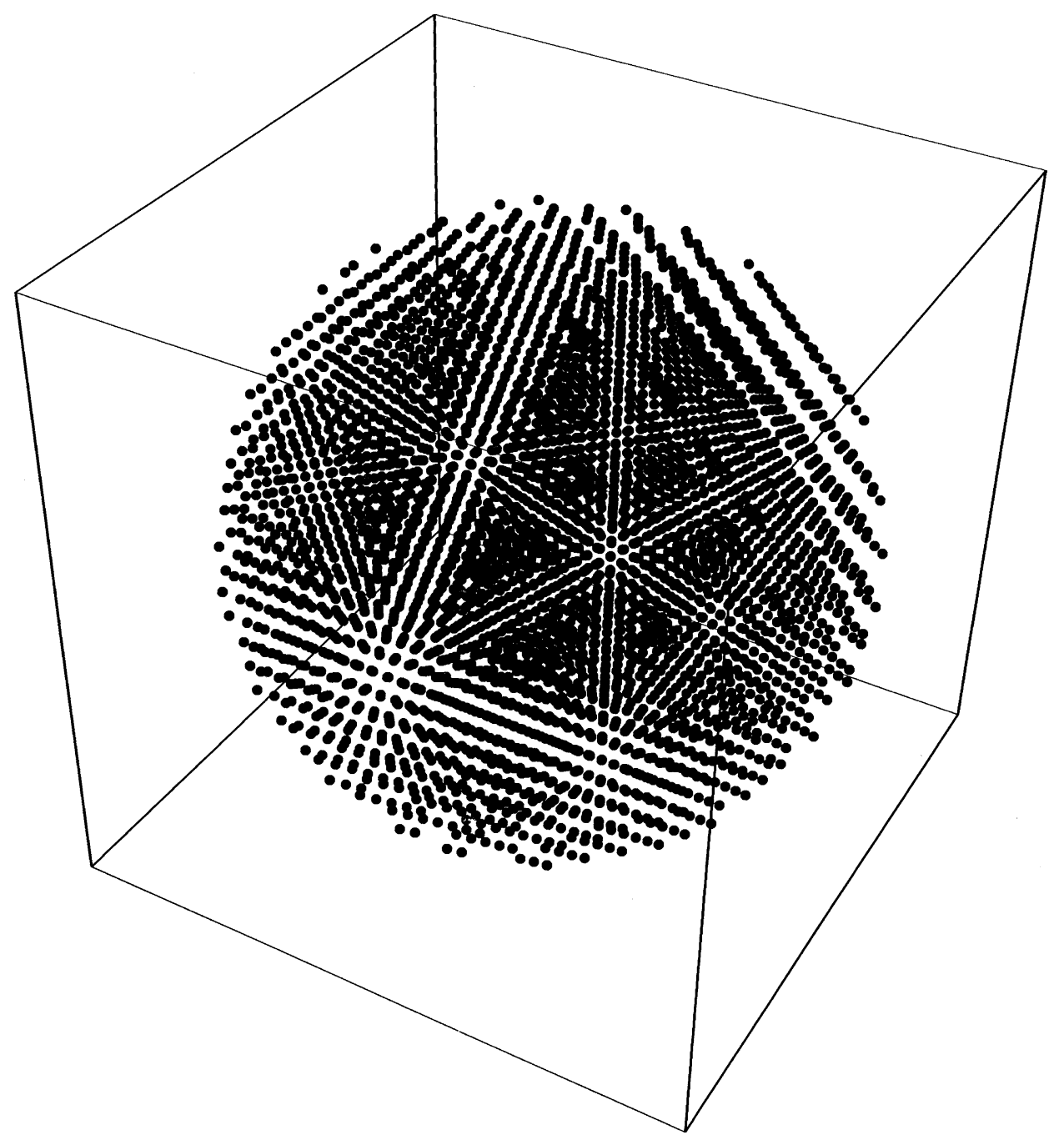

Fig. 7. Centers of dodecahedra formed into a rough-surfaced sphere with maximum diameter of 151 dodecahedra.

of the motor. For higher efficiency, the charge difference between the faces should be maximum when the dihedral angle is fully open and be reduced as the faces close, thereby reducing the kinetic energy imparted to the fluid squeezed out from between the plates.

\subsection{Power from the motor}

From Eq. (4), the dihedral angle between the two pentagon faces before the electric field is switched on to close them is

$\varphi=2 \pi-4 \arcsin \left[\frac{1}{2} \csc \left(\frac{\pi}{5}\right)\right] \simeq 2.21 \mathrm{rad}$.

The height of the pentagons from the edges where they join to the opposite vertex is

$h=\frac{l}{2}\left[\csc \left(\frac{\pi}{5}\right)+\cot \left(\frac{\pi}{5}\right)\right] \simeq 1.54 l$.

Neglecting the distance between the pentagons owing to thickness of the insulator, the distance between the pentagons at their furthest vertex is 


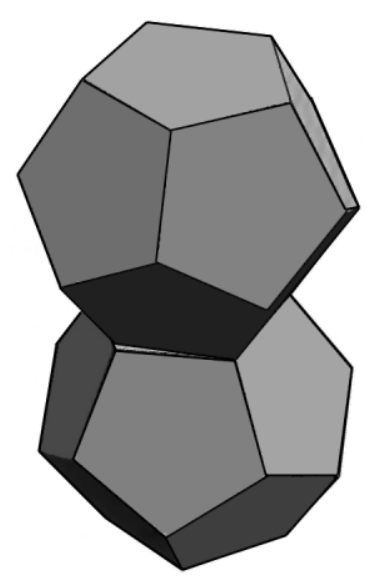

(a)

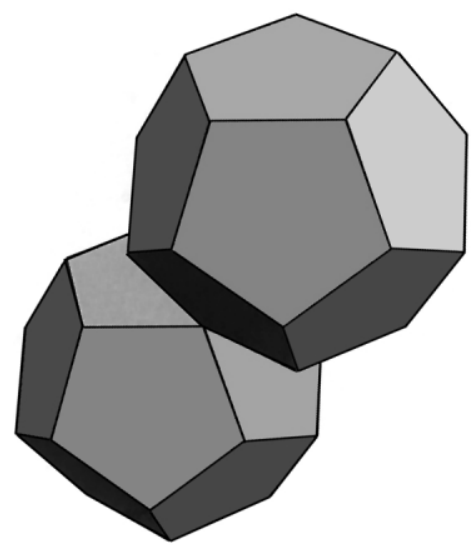

(c)

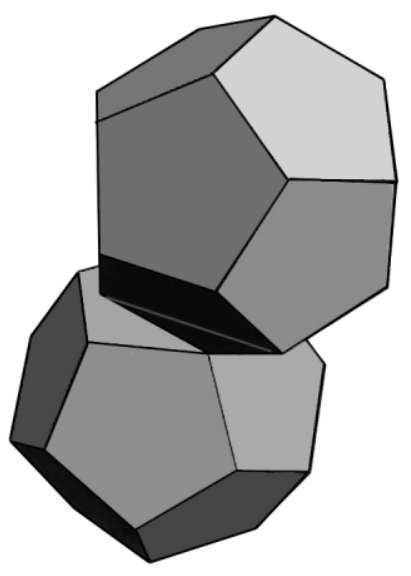

(b)

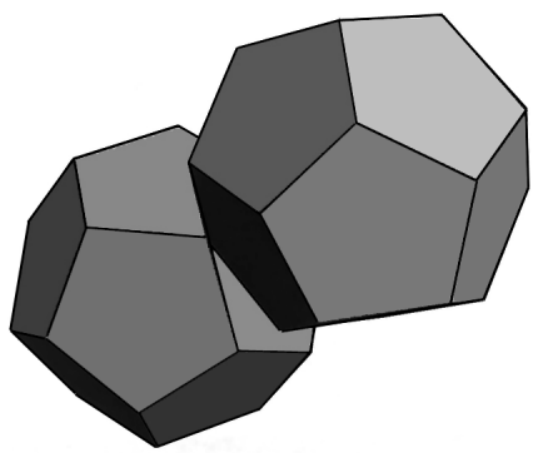

(d)

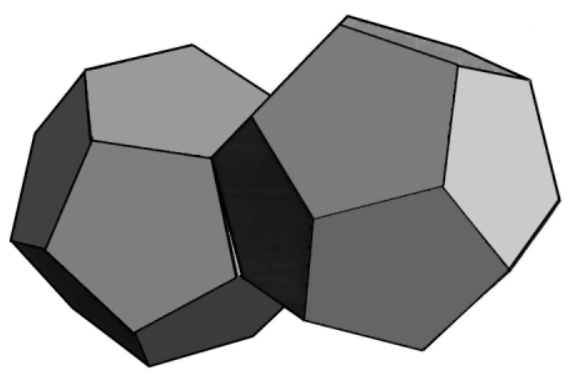

(e)

Fig. 8. Primitive motor: two micrites bound together with a '+' charge on face 11 of the lower micrite (specified micrite B) and a '-' charge on face 12 of the upper (specified micrite A). The charges on are then turned off and a ' + ' is applied to face 3 of micrite A, while a ' - ' is applied to face 5 of micrite B. Sequence is (a), (b), (c), (d), (e) as the micrites roll to there new positions. A repetition of the same pattern causes the two micrites to revolve around each other. 
$H=2 h \sin \left(\frac{\theta}{2}\right)$,

where $\theta$ is the dihedral angle between the pentagons at their common edge. The capacitance of the insulated pentagonal plates as a function of $\theta$ is a complicated integral, but can be approximated by

$$
\begin{aligned}
C(\theta) & \simeq \frac{5}{2}\left[\frac{\epsilon l \cot (\pi / 5) \csc (\theta / 2)}{\csc (\pi / 5)+\cot (\pi / 5)}\right] \\
& \simeq 1.12 \epsilon l \csc \left(\frac{\theta}{2}\right),
\end{aligned}
$$

which is singular at $\theta=0$ because we have neglected the insulation. The energy used in closure of the dihedral angle $\varphi$ to a minimum dihedral angle $\theta_{\mathrm{m}}$ is

$\Delta W=\frac{1}{2} \epsilon V^{2}\left[C\left(\theta_{\mathrm{m}}\right)-C(\varphi)\right]$.

Because of the viscosity of the fluid in which the micrites are immersed and the insulator on the conducting surfaces, the dihedral angle never completely closes, the conductors never come in contact. In a manner analogous to the derivation of Eq. (31), it can be shown that the angle between the pentagons at the common line is given as a function of time by

$\theta(t) \simeq \vartheta \exp \left[-\frac{2 \pi \epsilon V^{2} \tan (\pi / 5)}{15 \eta l^{2}} t\right]$.

Suppose the primitive motor were running at an angular velocity $\omega$. To complete a revolution it must approximately close the dihedral angle five times. So the time allowed for each approximate closure is

$t=\frac{2 \pi}{5 \omega}$

The minimum angle is therefore,

$\theta_{\mathrm{m}} \simeq \vartheta \exp \left[-\frac{4 \pi^{2} \epsilon V^{2} \tan (\pi / 5)}{75 \eta l^{2} \omega}\right]$.

For small enough $\omega$, the value of $\theta_{\mathrm{m}}$ could become arbitrarily small, making the $\Delta W$ as given by Eq. (60) indefinitely large. This is a result of the assumption that the thickness of the insulator on each pentagon is negligible.

\subsection{Power at small $\omega$}

When the viscosity is insufficient to prevent complete closure of the surfaces, we must consider the maximum capacitance resulting from the insulator thickness. This capacitance is

$C_{\text {ins }}=\frac{5 l^{2} \epsilon_{\text {ins }} \cot (\pi / 5)}{8 \delta}$,

where $\delta$ is the insulator thickness and $\epsilon_{\text {ins }}$ is the insulator permittivity. Taking $\epsilon_{\text {ins }}=\epsilon$, the energy of closure is

$$
\begin{aligned}
\Delta W & =\frac{1}{2} \epsilon V^{2}\left[C_{\mathrm{ins}}-C(\varphi)\right] \\
& \simeq \epsilon V^{2} l\left(0.430 \frac{l}{\delta}-0.911\right) .
\end{aligned}
$$

Using the example of a micrite with diametric face distance of $1 \mathrm{~mm}(l=0.449 \mathrm{~mm})$, insulator thickness of $1 \mu \mathrm{m}$, and face potential of $10 \mathrm{~V}(20 \mathrm{~V}$ interface potential), immersed in water with $\epsilon=7.18 \times 10^{-10}$, the energy of a single gap closure will be about 0.247863 ergs.

The angular velocity above which the gap does not completely close owing to viscosity can be estimated by setting $C\left(\theta_{\mathrm{m}}\right)=C_{\mathrm{ins}}$, which turns out to be

$$
\begin{aligned}
\omega_{\mathrm{m}}= & -\frac{4 \pi^{2} \epsilon V^{2} \tan (\pi / 5)}{75 \eta l^{2}} \\
& \times\left\{\ln \left[\frac{2}{\vartheta} \operatorname{arccsc}\left(\frac{5 l \cot (\pi / 5)}{8 k \delta}\right)\right]\right\}^{-1},
\end{aligned}
$$

where

$k=\frac{5}{2}\left[\frac{\cot (\pi / 5)}{\csc (\pi / 5)+\cot (\pi / 5)}\right]$.

The viscosity of pure water is approximately $\eta=$ $0.00101 \mathrm{~J} \mathrm{~s} \mathrm{~m}^{-3}$, so for the above described micrites we have $\omega_{\mathrm{m}}=90.7 \mathrm{~s}^{-1}$. The motor can, of course, run more slowly if the voltage is switched from face to face more slowly, but $\omega_{\mathrm{m}}$ is the highest angular velocity at which the faces will completely close on the insulator. The power generated by this motor is given by

$P=\frac{5}{2 \pi} \omega_{\mathrm{m}} \Delta W=1.79 \mu \mathrm{W}$.

\subsection{Power at large $\omega$}

If the motor rotates at an angular velocity greater than $\omega_{\mathrm{m}}$, the gaps between the pentagonal faces of the two dodecahedra will not completely close. There will also be some floating motion where the two 
dodecahedra actually lose contact. If we assume, however, that the common line of contact is still a good approximation, we can calculate the power for cases where viscosity prevents complete closure of the gaps. Evaluating Eq. (60) directly by using Eqs. (59) and (63), it can be shown that

$$
\begin{aligned}
P= & \frac{25 l \omega \epsilon V^{2} \cot (\pi / 5)}{8 \pi[\csc (\pi / 5)+\cot (\pi / 5)]} \\
& \times\left\{\csc \left[\frac{\vartheta}{2} \exp \left(-\frac{4 \pi^{2} \epsilon V^{2} \tan (\pi / 5)}{75 \eta l^{2} \omega}\right)\right]\right. \\
& \left.-\csc \left(\frac{\vartheta}{2}\right)\right\} .
\end{aligned}
$$

Again, this expression is singular at $\omega=0$ because it does not account for the insulator and would allow the capacitance between faces to become indefinitely large. At $\omega=90.7 \mathrm{~s}^{-1}$, Eq. (69) gives $P=1.79 \mu \mathrm{W}$, which is in agreement with Eq. (68) to three figures of accuracy.

For very large values of $\omega$, Eq. (69) monotonically asymptotes to

$$
P \simeq \frac{\pi \epsilon^{2} V^{4} \vartheta \cot (\vartheta / 2) \csc (\vartheta / 2)}{12 \eta l[\csc (\pi / 5)+\cot (\pi / 5)]}
$$

For the numerical example we are considering, this corresponds to $P \simeq 0.0192 \mu \mathrm{W}$. In other words, the net power of the motor decreases if it is run so fast as to prevent closure of the faces. Therefore, it is strongly indicated that $1.79 \mu \mathrm{W}$, as given by Eq. (68), is the greatest power generated by this two-micrite system immersed in water.

\subsection{Adding more micrites to the motor}

A third micrite (specified micrite C) could be attached to micrite $\mathrm{B}$ diametrically from micrite A. The end micrites will them orbit around micrite B if all are given the same command sequence as shown in Table 2. An indefinite number of micrites may be added to form a writhing linear filament. No propulsion will occur because all the opening and closing on gaps as shown in Figs. 8(a)-8(d) are symmetrical. The filament will only churn the fluid around it.

Now consider what happens if the electric dipole is not across diametric faces of the micrite but is out of phase by one face. To be specific, the configuration looks like this.
Table 2

Time sequence of charges on faces of three dodecahedral micrites

\begin{tabular}{rrrrrrr}
\hline$t$ & $\mathrm{~A}_{-}$ & $\mathrm{A}_{+}$ & $\mathrm{B}_{-}$ & $\mathrm{B}_{+}$ & $\mathrm{C}_{-}$ & $\mathrm{C}_{+}$ \\
\hline 0 & 3 & 4 & 3 & 4 & 3 & 4 \\
1 & 5 & 6 & 5 & 6 & 5 & 6 \\
2 & 7 & 8 & 7 & 8 & 7 & 8 \\
3 & 9 & 10 & 9 & 10 & 9 & 10 \\
4 & 11 & 12 & 11 & 12 & 11 & 12 \\
$\vdots$ & $\vdots$ & $\vdots$ & $\vdots$ & $\vdots$ & $\vdots$ & $\vdots$ \\
\hline
\end{tabular}

$\mathrm{A}_{+} \quad \mathrm{A}_{-} \quad \mathrm{B}_{+} \quad \mathrm{B}_{-} \quad \mathrm{C}_{+} \quad \mathrm{C}_{-} \quad \mathrm{D}_{+} \quad \mathrm{D}_{-} \quad \mathrm{E}_{+} \quad \mathrm{E}_{-}$ $\begin{array}{llllllllll}11 & 4 & 11 & 4 & 11 & 4 & 11 & 4 & 11 & 4\end{array}$

Propulsion can only occur if the symmetry of movement is somehow broken. This implies independent commands to more than one type of micrite. Micrites must be independently addressable in at least two classes.

\section{Propulsion by sinuous motion of joined dodecahedra}

Suppose we use the following rule to join the faces of dodecahedra. The contact faces are sequential: face 1 of dodecahedron A contacts face 2 of dodecahedron $\mathrm{B}$, face 3 of dodecahedron $\mathrm{B}$ contacts face 4 of dodecahedron $\mathrm{C}$, face 5 of dodecahedron $\mathrm{C}$ contacts face 6 of dodecahedron $\mathrm{D}$, etc. The dihedral angles between dodecahedra are sequential: the dihedral angles made at the first interface will consist of the following faces: $3: 4,5: 6,7: 8,9: 10,11: 12$. For all interfaces except the interface between dodecahedron $A$ and dodecahedron $\mathrm{B}$ (called AB), two of the dihedrals are sequential but in reversed order. For example, the interface $\mathrm{BC}$ has dihedrals with faces 8:7 and 10:9.

\subsection{Contact and orientation}

The above-described assembly algorithm will produce a cycle that repeats every 6 interfaces. Table 3 summarizes the contact and orientation at each of the 6 interfaces of 7 dodecahedra specified A through G.

In Table 3, the faces shown in bold are in contact, the normal font are the faces forming dihedrals. At interfaces 2 through 6 , two of the dihedrals are numbered sequentially but in backward order. These are shown in italics in the table. 
Table 3

Interface structure of dodecahedral micrites bound at sequential faces and oriented with sequential dihedrals

\begin{tabular}{llllll}
\hline $\mathrm{AB}$ & $\mathrm{BC}$ & $\mathrm{CD}$ & $\mathrm{DE}$ & $\mathrm{EF}$ & $\mathrm{FG}$ \\
\hline $\mathbf{1 : 2}$ & $1: 2$ & $1: 2$ & $1: 2$ & $1: 2$ & $1: 2$ \\
$3: 4$ & $\mathbf{3 : 4}$ & $3: 4$ & $4: 3$ & $4: 3$ & $3: 4$ \\
$5: 6$ & $5: 6$ & $\mathbf{5 : 6}$ & $5: 6$ & $6: 5$ & $6: 5$ \\
$7: 8$ & $8: 7$ & $7: 8$ & $\mathbf{7 : 8}$ & $7: 8$ & $8: 7$ \\
$9: 10$ & $10: 9$ & $10: 9$ & $9: 10$ & $\mathbf{9 : 1 0}$ & $9: 10$ \\
$11: 12$ & $11: 12$ & $12: 11$ & $12: 11$ & $11: 12$ & $\mathbf{1 1 : 1 2}$ \\
\hline
\end{tabular}

The sequence described in Table 3 consists of two triples of the form shown in Fig. 9. These are joined at a $127^{\circ}$ angle to form the sextuple described in Table 3 and illustrated in Fig. 10. Fig. 11 shows a sequence of
9 consisting of 3 triples and Fig. 12 shows a sequence of 12 consisting of 4 triples the total sinuous geometric cycle. The next cycle is simply the mirror image. If we connect a set of 14 starting with the 1:2 interface, we get the configuration shown in Fig. 13. Starting in the upper left corner of Fig. 13, we have the following sequence of 13 interfaces:

$\begin{array}{llllll}1: 2 & 3: 4 & 5: 6 & 7: 8 & 9: 10 & 11: 12 \\ 1: 2 & 3: 4 & 5: 6 & 7: 8 & 9: 10 & 11: 12 \\ 1: 2 & & & & & \end{array}$

The configuration has a parity conserving plane of symmetry through the middle 11:12 interface (between the $\mathrm{G}$ and $\mathrm{H}$ dodecahedra).

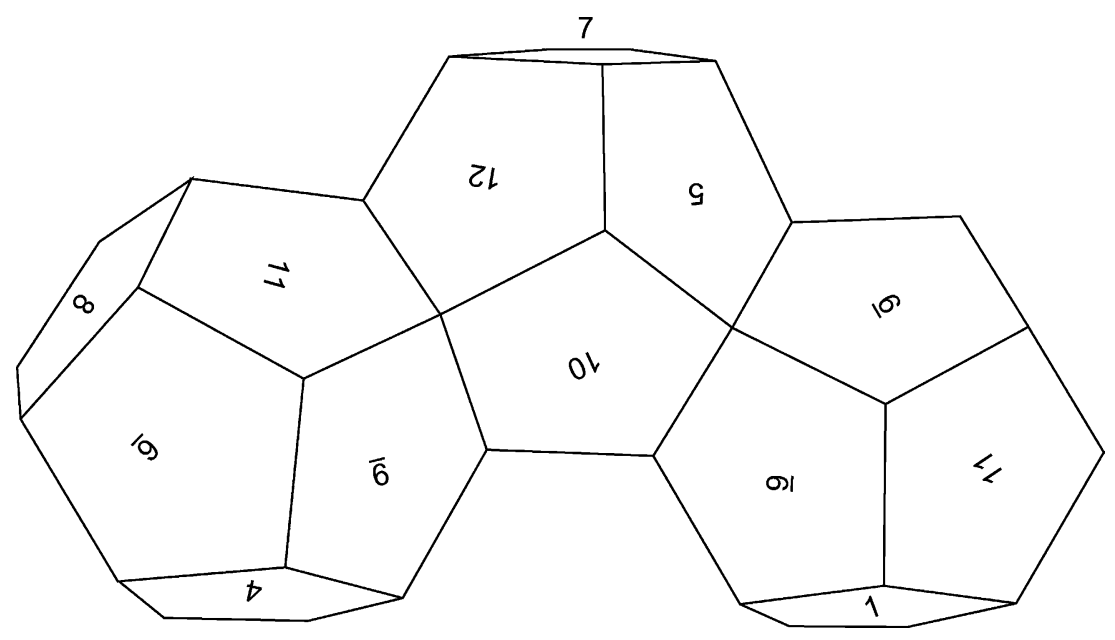

Fig. 9. Micrite triple.

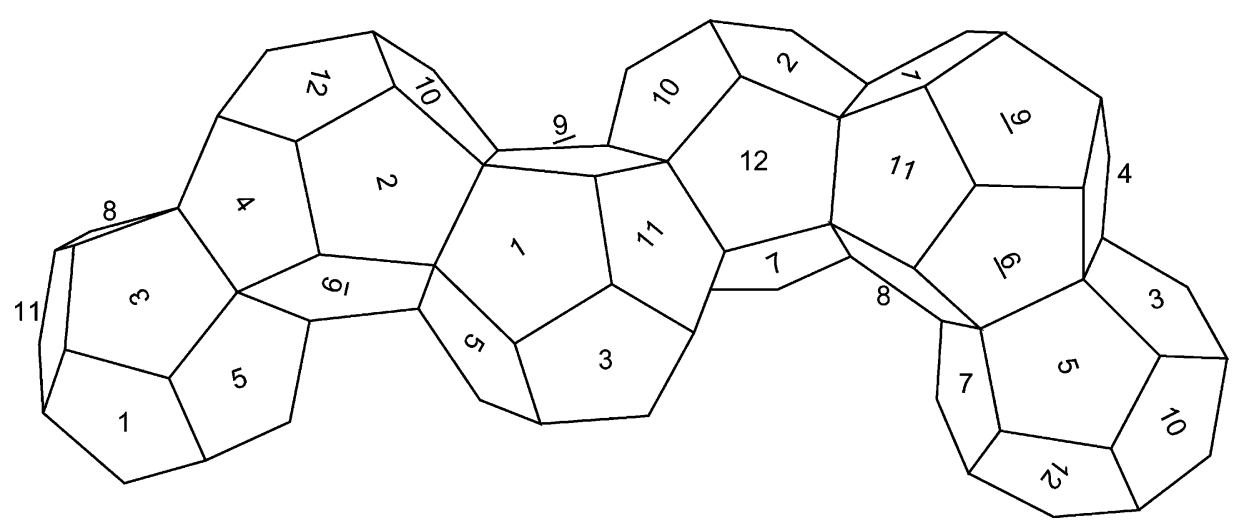

Fig. 10. Sequence in Table 3 consists of two triples, joined at a $127^{\circ}$ angle to form the sextuple. 


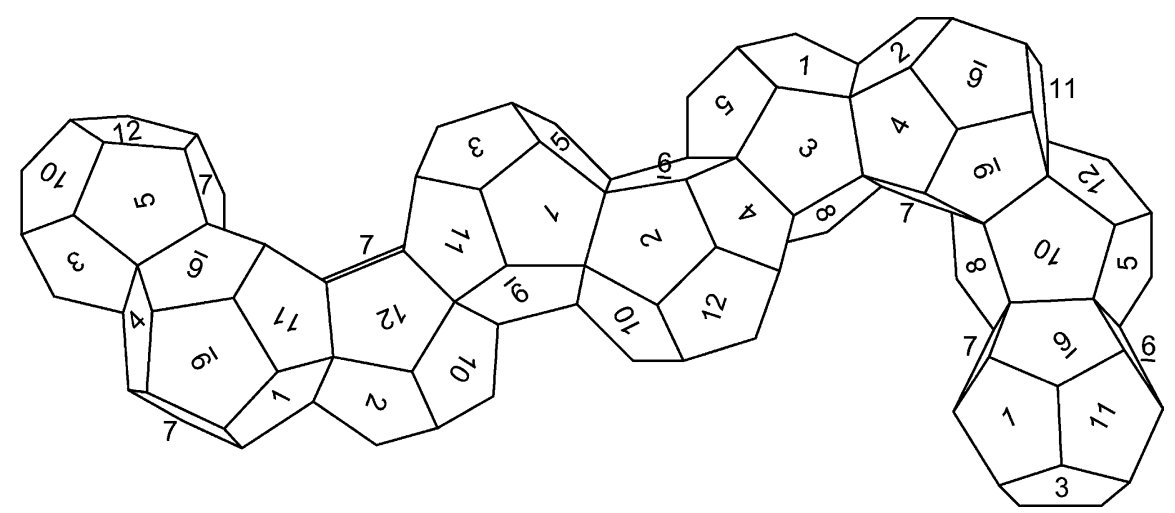

Fig. 11. Sequence of 9 consisting of 3 triples.

\subsection{Sinuous motion}

Now issue a single command for the micrites to move charge so they close the next lower numbered pair of pentagons. So the interface 5:6 becomes 3:4, and $1: 2$ becomes $11: 12$, etc. The new-configuration sequence of interfaces becomes:

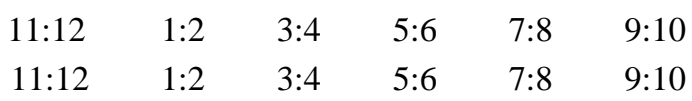

$11: 12$ and the new configuration is shown in Fig. 14. Note that although each dodecahedron only rotates to a new face, the new configuration is as if a micrite were taken off the right end and re-attached at the appropriate sequential face at the left end. Also note from Table 3, the pentagon surfaces that close are always in sequential order, never in backward sequential order. For example, 1:2 closes on 3:4, never on $4: 3$, similarly $3: 4$ closes on $5: 6$, never on $6: 5$, etc. This has the simplifying result that all the electric dipoles remain

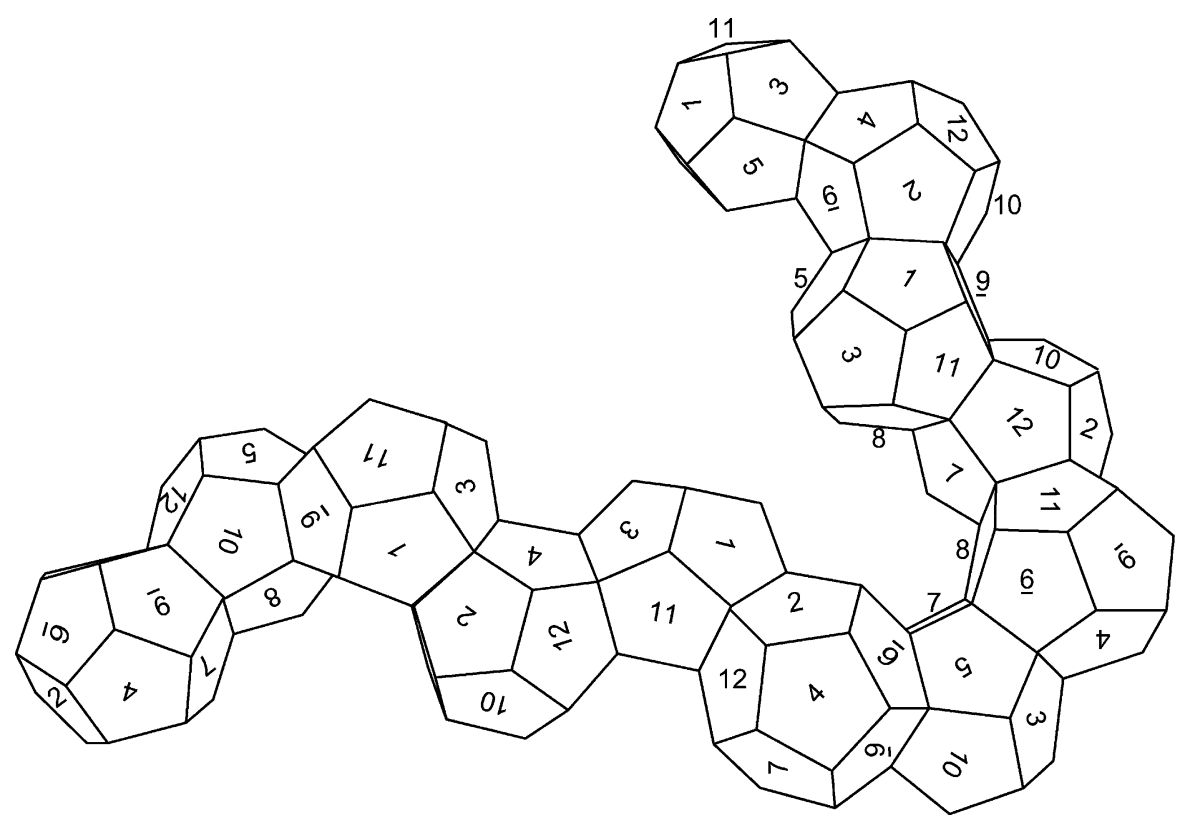

Fig. 12. Sequence of 12 consisting of 4 triples, the total sinuous geometric cycle. 


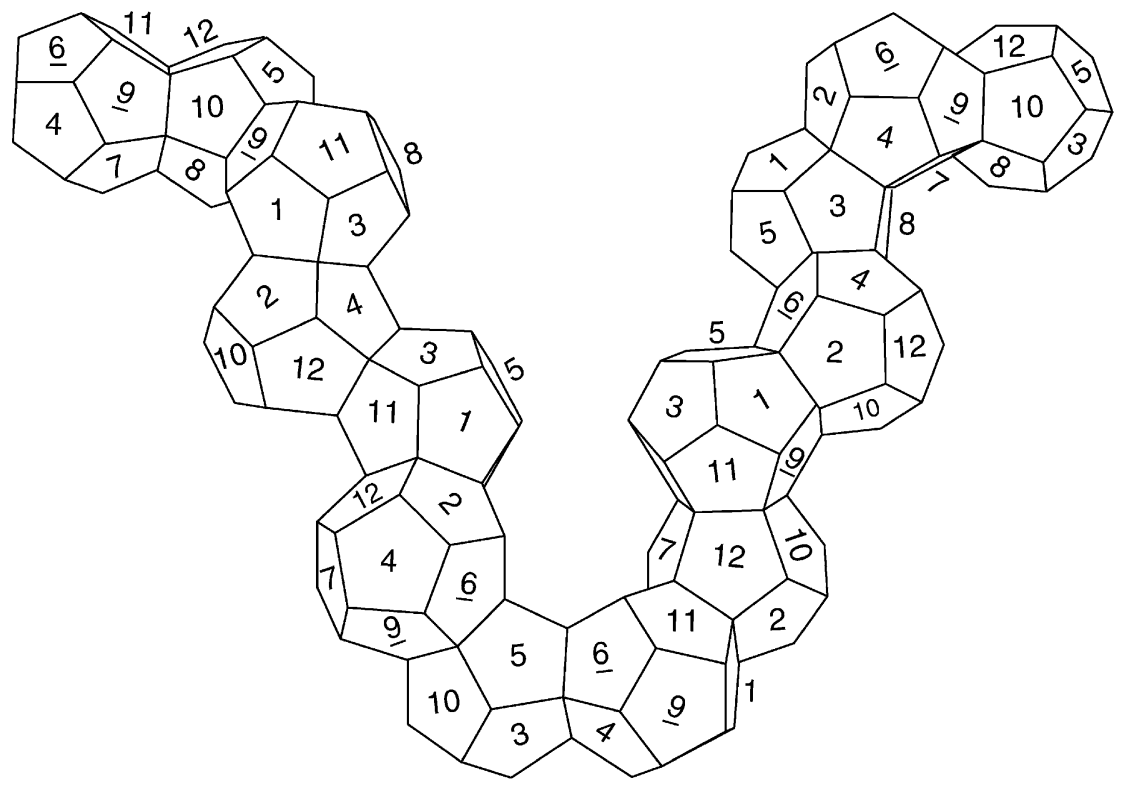

Fig. 13. Configuration of a set of 14 micrites starting with the $1: 2$ interface.

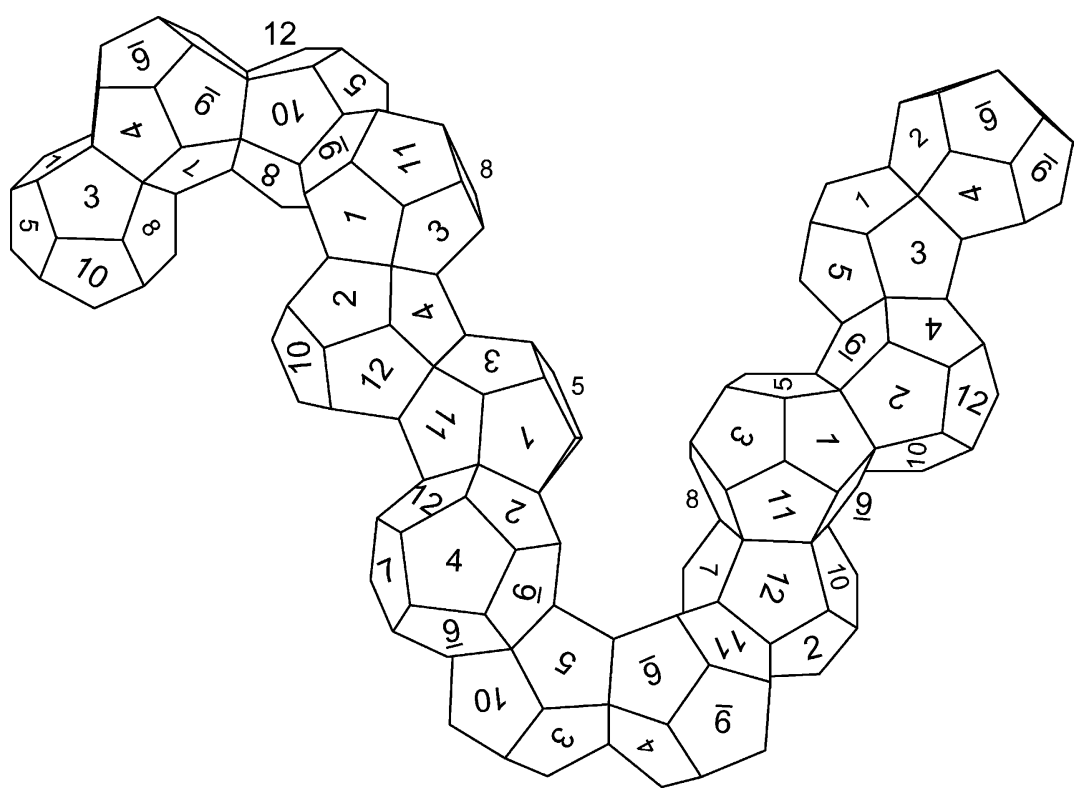

Fig. 14. Configuration resulting from a single command for the micrites to move charge so they close the next lower numbered pair of pentagons, and the interface 5:6 becomes 3:4, and 1:2 becomes 11:12, etc. Although each dodecahedron only rotates to a new face, the new configuration is as if a micrite were taken off the right end and re-attached at the appropriate sequential face at the left end. 


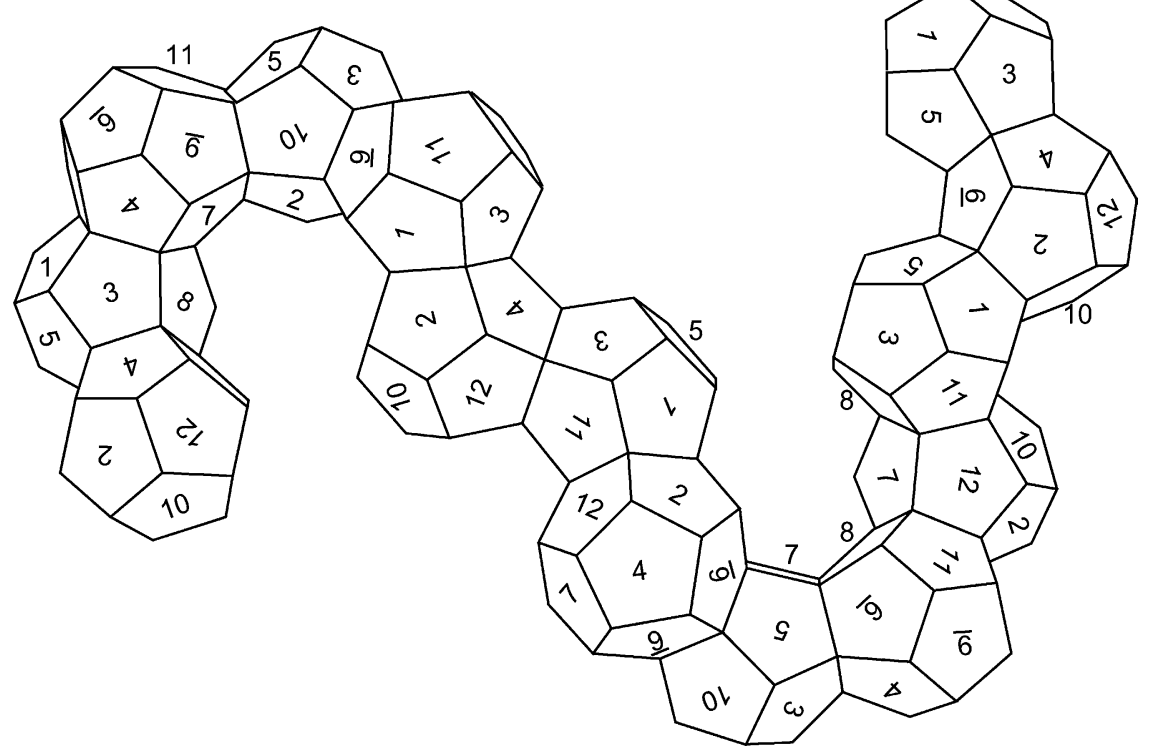

Fig. 15. Configuration after the same command is given to all the micrites again.

in the same direction as the numerical sequential ordering.

Fig. 15 shows the configuration after the same command is given to all the micrites again. Again it appears that the micrite at the right end of the chain has been moved to the left end.

Fig. 16 shows the entire set of seven rotations that will convert the chain of 14 micrites from the initial configuration shown in Fig. 13 to its vertical inverse shown in Fig. 16(g). The vertical inverse is also the same as if Fig. 16(a) [same as Fig. 13] had been turned upside-down with a rotation of $\pi$ about a horizontal axis. Fig. 17 is an enlargement of this configuration. Table 4 shows the sequence of binding interfaces for the cycle to inversion.

The time sequence of charges on the pentagon faces that will produce the sinuous propulsion pattern is shown in Table 5.

The algorithm for charging each face is then

$F_{ \pm}=\lfloor(n+t) \bmod 6\rfloor+\frac{5}{2} \pm \frac{1}{2}$,

where $n$ indicates the number of the micrite. Because of the selection of end point and phase, micrite A is specified by $n=-1$, micrite $\mathrm{B}$ is specified by $n=0$, micrite $\mathrm{C}$ is specified by $n=1$, micrite $\mathrm{D}$ is specified by $n=2$, etc. For the complexity of the sinuous motion, this is a remarkably simple algorithm. It does, however, require some micrite-to-micrite communication so each can know its number in the sequence.

A greater thrust and efficiency is obtained by binding together several cycles to resemble a biological flagellum, as shown in Fig. 18.

\subsection{Power expended in micrite rolling}

The power consumed in sinuous motion is similar to the previously described primitive motor. The essential difference is the micrites undergoing sinuous motion repeat a cycle of rolling over six faces as indicated by the algorithm described by Eq. (71), as opposed to the primitive motor completing a cycle by rolling over 5 faces as indicated by the algorithm described by Eq. (55). A total of 12 face closures will be required for a complete cycle of the chain to its original configuration. The power is analogous to Eq. (68), and if there are $N$ micrites in the chain, the power will be

$P=\frac{3 \epsilon V^{2}}{\pi}\left[C\left(\theta_{\mathrm{m}}\right)-C(\varphi)\right] N \Omega$,

where $2 \pi / \Omega$ is the time taken for the chain to resume its original configuration. For our standard $1 \mathrm{~mm}$ micrites in oil, the highest angular velocity at which 


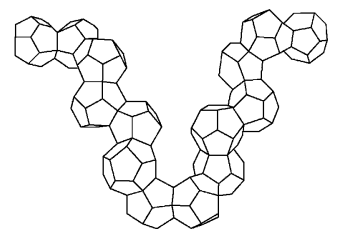

a

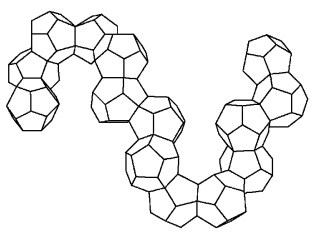

c

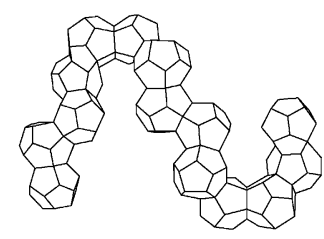

e

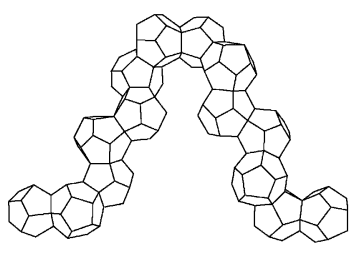

$\mathrm{g}$

Fig. 16. Entire set of seven rotations that will convert the chain of 14 micrites from the initial configuration shown in Fig. 13 to its vertical inverse shown in $(\mathrm{g})$. The vertical inverse is also the same as if it had been turned upside-down with a rotation of $\pi$ about a horizontal axis.

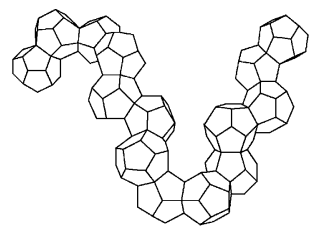

b

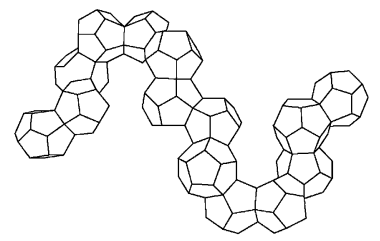

d

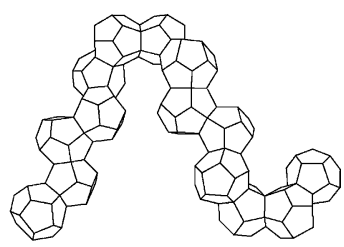

f the faces will completely close on the insulator is $\Omega=37.8 \mathrm{~s}^{-1}$. The electrical power dissipated in the sinuous motion is

$P=1.79 N \mu \mathrm{W}$,

to be compared with Eq. (68).

\subsection{Propulsion}

Our sinuous assembly of $1 \mathrm{~mm}$ micrites immersed in a viscous fluid will still have Reynolds number sufficiently small to exhibit Aristotlean or "Stokesian" behavior. Flagellum-propelled organisms have already been well studied [7-10] and a fine review is given by Azuma [11].

Following Azuma, I take the shape to be truly sinusoidal, so in the frame of the micrite chain,

$y=a \sin \left[\frac{2 \pi}{\lambda}(x+c t)\right]$,

where $a$ is the amplitude, $\lambda$ is the wavelength, and $c$ is the wave propagation speed. The shape of the micrite configuration is not exactly sinusoidal, but neither are biological flagella [12]. Similarly, the micrites are not all in the same plane, but biological flagella are not perfectly planar either [13]. But in all cases, the motion is well approximated by a moving sine wave as given in Eq. (74).

The value of $c$ may be positive or negative, depending on direction of motion. In a reference frame moving at velocity equal to that of an element of the micrite chain, the relative fluid velocity normal and tangential to an element of the micrite chain is

$$
\begin{aligned}
& u_{\mathrm{N}}=(\dot{y}-v) \cos \theta+(u-\dot{x}) \sin \theta \\
& u_{\mathrm{T}}=-(\dot{y}-v) \sin \theta+(u-\dot{x}) \cos \theta,
\end{aligned}
$$

Table 4

\begin{tabular}{|c|c|c|c|c|c|c|c|c|c|c|c|c|}
\hline $\mathrm{AB}$ & $\mathrm{BC}$ & $\mathrm{CD}$ & DE & $\mathrm{EF}$ & FG & GH & HI & IJ & $\mathrm{JK}$ & KL & LM & $\mathrm{MN}$ \\
\hline $1: 2$ & $3: 4$ & $5: 6$ & $7: 8$ & $9: 10$ & $11: 12$ & $1: 2$ & $3: 4$ & $5: 6$ & $7: 8$ & $9: 10$ & $11: 12$ & $1: 2$ \\
\hline $11: 12$ & $1: 2$ & $3: 4$ & $5: 6$ & $7: 8$ & $9: 10$ & $11: 12$ & $1: 2$ & $3: 4$ & $5: 6$ & $7: 8$ & $9: 10$ & $11: 12$ \\
\hline $9: 10$ & $11: 12$ & $1: 2$ & $3: 4$ & $5: 6$ & $7: 8$ & $9: 10$ & $11: 12$ & $1: 2$ & $3: 4$ & $5: 6$ & $7: 8$ & $9: 10$ \\
\hline 7:8 & $9: 10$ & $11: 12$ & $1: 2$ & $3: 4$ & $5: 6$ & $7: 8$ & $9: 10$ & $11: 12$ & $1: 2$ & $3: 4$ & $5: 6$ & $7: 8$ \\
\hline $5: 6$ & $7: 8$ & $9: 10$ & $11: 12$ & $1: 2$ & $3: 4$ & $5: 6$ & $7: 8$ & $9: 10$ & $11: 12$ & $1: 2$ & $3: 4$ & $5: 6$ \\
\hline $3: 4$ & $5: 6$ & $7: 8$ & $9: 10$ & $11: 12$ & $1: 2$ & $3: 4$ & $5: 6$ & $7: 8$ & $9: 10$ & $11: 12$ & $1: 2$ & $3: 4$ \\
\hline $1: 2$ & $3: 4$ & $5: 6$ & $7: 8$ & $9: 10$ & $11: 12$ & $1: 2$ & $3: 4$ & $5: 6$ & $7: 8$ & $9: 10$ & $11: 12$ & $1: 2$ \\
\hline
\end{tabular}

Sequence of binding interfaces for half cycle of sinuous motion shown in Fig. 16 


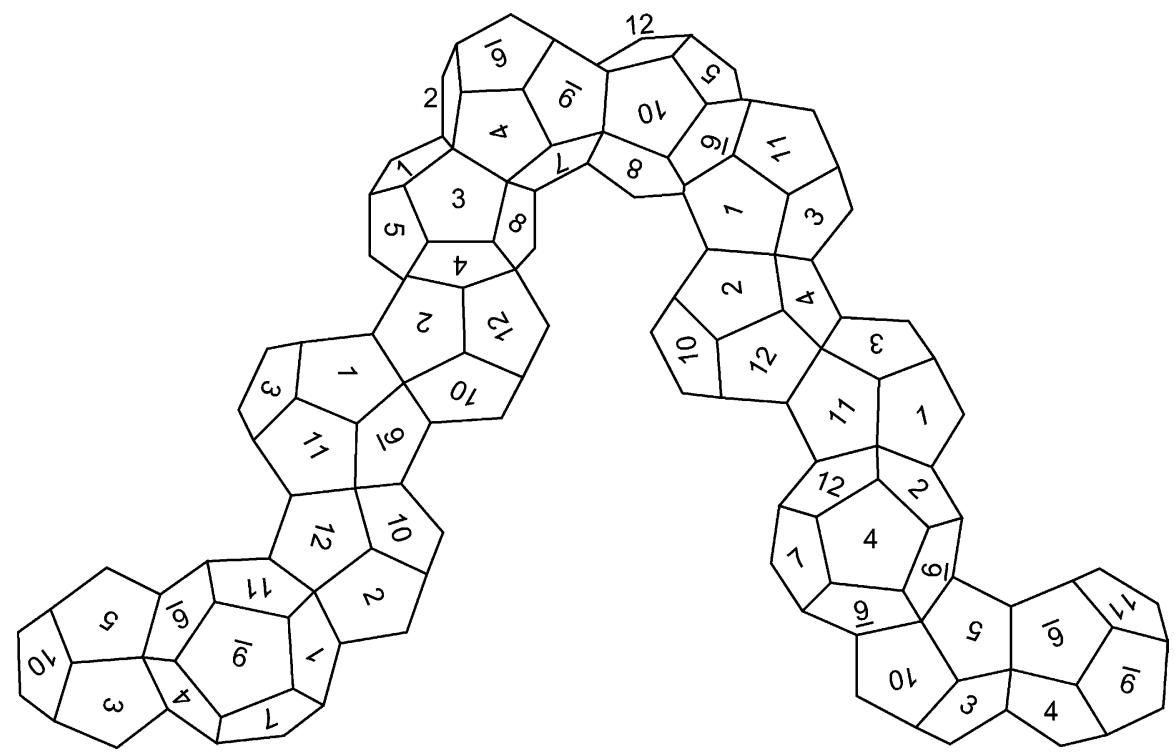

Fig. 17. Enlargement of this configuration shown in Fig. 16(g). Table 4 shows the sequence of binding interfaces for the cycle to inversion.

Table 5

Time sequence of charges on faces of micrites in indefinite chain undergoing sinuous motion

\begin{tabular}{|c|c|c|c|c|c|c|c|c|c|c|c|c|c|c|c|c|c|c|c|}
\hline$t$ & $\mathrm{~A}_{-}$ & $\mathrm{A}_{+}$ & $\mathrm{B}_{-}$ & $\mathrm{B}_{+}$ & $\mathrm{C}_{-}$ & $\mathrm{C}_{+}$ & $\mathrm{D}_{-}$ & $\mathrm{D}_{+}$ & $\mathrm{E}_{-}$ & $\mathrm{E}_{+}$ & $\mathrm{F}_{-}$ & $\mathrm{F}_{+}$ & $\mathrm{G}_{-}$ & $\mathrm{G}_{+}$ & $\mathrm{H}_{-}$ & $\mathrm{H}_{+}$ & $\mathrm{I}_{-}$ & $\mathrm{I}_{+}$ & $\ldots$ \\
\hline 0 & 12 & 1 & 2 & 3 & 4 & 5 & 6 & 7 & 8 & 9 & 10 & 11 & 12 & 1 & 2 & 3 & 4 & 5 & $\ldots$ \\
\hline 1 & 2 & 3 & 4 & 5 & 6 & 7 & 8 & 9 & 10 & 11 & 12 & 1 & 2 & 3 & 4 & 5 & 6 & 7 & $\ldots$ \\
\hline 2 & 4 & 5 & 6 & 7 & 8 & 9 & 10 & 11 & 12 & 1 & 2 & 3 & 4 & 5 & 6 & 7 & 8 & 9 & $\ldots$ \\
\hline 3 & 6 & 7 & 8 & 9 & 10 & 11 & 12 & 1 & 2 & 3 & 4 & 5 & 6 & 7 & 8 & 9 & 10 & 11 & $\ldots$ \\
\hline 4 & 8 & 9 & 10 & 11 & 12 & 1 & 2 & 3 & 4 & 5 & 6 & 7 & 8 & 9 & 10 & 11 & 12 & 1 & $\ldots$ \\
\hline 5 & 10 & 11 & 12 & 1 & 2 & 3 & 4 & 5 & 6 & 7 & 8 & 9 & 10 & 11 & 12 & 1 & 2 & 3 & $\ldots$ \\
\hline$\vdots$ & $\vdots$ & $\vdots$ & $\vdots$ & $\vdots$ & $\vdots$ & $\vdots$ & $:$ & $\vdots$ & $\vdots$ & : & : & $:$ & & r. & $\cdot$ & $\vdots$ & : & $\vdots$ & $\vdots$ \\
\hline
\end{tabular}

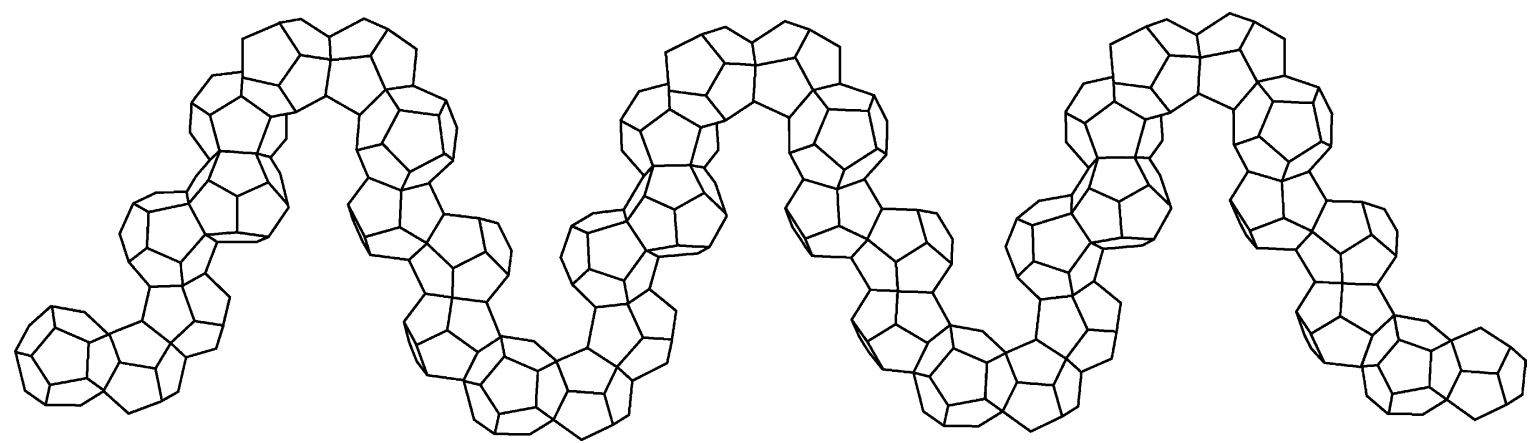

Fig. 18. A greater thrust and efficiency is obtained by binding together several cycles to resemble a biological flagellum. 
where $\theta$ is the pitch angle in the vicinity of the element, recognizing that on the micrite scale, the chain is not smooth and the interaction with the fluid is complicated by the opening and closing of gaps between pentagons. Since the Reynolds number is small, end effects are small and the drag is proportional to the velocity.

\subsection{Swimming speed}

The drag forces acting on the micrite chain can be approximated by

$\frac{\mathrm{d} D_{\mathrm{N}}}{\mathrm{d} s}=\frac{1}{2} \rho d c_{\mathrm{N}} u_{\mathrm{N}}, \quad \frac{\mathrm{d} D_{\mathrm{T}}}{\mathrm{d} s}=\frac{1}{2} \rho d c_{\mathrm{T}} u_{\mathrm{T}}$,

where $s$ refers to distance along the chain, $D_{\mathrm{N}}$ the normal drag, $D_{\mathrm{T}}$ the tangential drag, $c_{\mathrm{N}}$ and $c_{\mathrm{T}}$ are the normal and tangential modified drag coefficients, and $d$ is the cylindrical equivalent of the chain diameter, about $1 \mathrm{~mm}$ for our example. If we take [10]

$\frac{c_{\mathrm{T}}}{c_{\mathrm{N}}} \simeq \frac{1}{2}$,

then, assuming the wave propagation speed $c$ is positive, the forward thrust is

$F \simeq \frac{l c_{\mathrm{N}}}{2 \lambda^{2}}\left(2 \pi^{2} a^{2} c-\lambda^{2} u-4 \pi^{2} a^{2} u\right)$,

where $l$ is the total length of the micrite chain. This is the thrust that the micrite chain can apply to an object it is trying to move. That object could be an apparatus constructed of other micrites and its drag will be derived to be some variant of Stokes' law in the low Reynolds number regime. If there is no "head" on the "flagellum", the speed of the micrite chain is given by setting $F=0$ in Eq. (78) from which we obtain

$u=\frac{2 \pi^{2} a^{2}}{\lambda^{2}+4 \pi^{2} a^{2}} c$,

which is independent of drag coefficients or length. For our $1 \mathrm{~mm}$ micrite formed in a chain as shown in Fig. 16, $a \sim 0.43 \mathrm{~cm}, \lambda \sim 0.40 \mathrm{~cm}$, and $c=\lambda \Omega / 2 \pi$. The maximum power is produced when the gaps are not prevented from closing by the viscosity. In this case,

$\Omega=\frac{5}{12} \omega_{\mathrm{m}}=37.8 \mathrm{~s}^{-1}$, where $\omega_{\mathrm{m}}=90.7 \mathrm{~s}^{-1}$ is given by Eq. (66), and the factor of $5 / 12$ derives from a complete cycle involving 12 face closures rather than 5 as for the primitive motor. This results in a wave propagation speed of $c=2.43 \mathrm{~cm} \mathrm{~s}^{-1}$, which from Eq. (79) results in a swimming speed of $u=1.19 \mathrm{~cm} \mathrm{~s}^{-1}$ in pure water.

\section{Summary conclusions}

This paper has been a brief look at self-organizing micrites in three dimensions. The Platonic solids were chosen because their faces are all regular pentagons and they can bind together with perfect overlap. The perfect overlap provides for maximum binding strength for applied charge and minimal stray fields. This would not be possible for the Archimedian solids, e.g. whose faces are regular polygons but not all the same.

Among the Platonic solids, only the cube and dodecahedron can fill all space leaving no voids. And between these two, only the dodecahedron is capable of a broad range of motions because its dihedral angle with others of its species is less than $\pi$.

I have analyzed the dynamics of Platonic solids in the process of assembly into structures, and have derived the time scales for: (1) terminal convergence; (2) translation and alignment; and (3) distant convergence. For translation and alignment of faces moving across each other, I have shown that a lubricant is necessary, which will be the fluid in which the micrites are immersed, and may endow the micrites with the collateral benefit of neutral buoyancy. Use of the lubricant forces the dynamics into the low-Reynolds-number regime. Immersion in air to achieve the same effect is not dismissed, but requires a micrite size scale far less than $1 \mathrm{~mm}$ to attain the requisite Reynolds number.

I have shown a simple algorithm for making a primitive two-component motor and a surprisingly simple algorithm for making a chain of micrites undergo sinuous motion resulting in self-propulsion. The chain can provide thrust to move other structures as well as propelling itself.

There are undoubtedly many other algorithms of more complexity, which would result in more complex agglomerations and more complex motions. 


\section{References}

[1] J.C. Solem, On the motility of military microrobots, Los Alamos National Laboratory Report LA-12133(U), 1991.

[2] J.C. Solem, On the motility of microrobots, in: C. Langton, (Ed.), Artificial Life III, SFI Studies in the Sciences of Complexity, Addison-Wesley, Reading, MA, 1994, pp. 359-380.

[3] Schläfli, Theorie der veilfachen Kontinuität, Denkschriften der Schweizerischen naturforschenden Gesellschaft 38 (1901).

[4] H.S.M. Coxeter, Regular Polytopes, Dover, New York, 1973, p. 5.

[5] J.A. Williams, Engineering Tribology, Oxford University Press, Oxford, 1996, pp. 200-231.

[6] N. Bowden, A. Terfort, J. Carbeck, G. Whitesides, Selfassembly of mesoscale objects into ordered two-dimensional arrays, Science 276 (1997) 233.

[7] G. Taylor, Analysis of the swimming of microscopic organisms, Proc. Roy. Soc. Lond. A 209 (1951) 447-461.

[8] G. Taylor, The action of waving cylindrical tails in propelling microscopic organisms, Proc. Roy. Soc. Lond. A 211 (1952) 225-239.

[9] G.H. Hancock, Self-propulsion of microscopic organisms through liquids, Proc. Roy. Soc. Lond. (Biol.) 217 (1953) 96-121.

[10] J. Lighthill, Flageller hydrodynamics, SIAM Rev. 18 (1975) 161-230.

[11] A. Azuma, Swimming by snaking, in: The Biokinetics of Flying and Swimming, Springer, Tokyo, 1992 (Chapter 5).

[12] C.J. Brokaw, Non-sinusoidal bending waves of sperm flagella, J. Exp. Biol. 43 (1965) 155-169.

[13] H.C. Berg, How spirochetes may swim, J. Theoret. Biol. 56 (1976) 269-273.

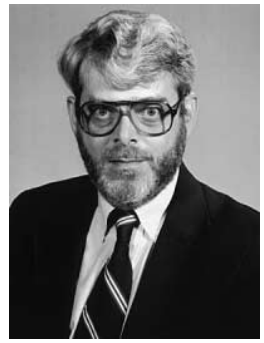

Johndale C. Solem is a Fellow of Los Alamos National Laboratory. He received his BS (1963), MS (1965), M Phil (1967), and $\mathrm{PhD}$ (1968) from Yale University, and joined the Theoretical Division of Los Alamos in 1969, after a brief tenure as a Research Associate at Yale. His research activities have included both theory and experiment in the areas of: magnetism; particle and radiation transport; plasma physics; nuclear physics; nuclear explosive theory; equations-ofstate; artificial intelligence and robotics; computational science; $\mathrm{X}$-ray microholography; antiproton science and technology; mathematical physics; astrophysic; exotic methods of spacecraft propulsion; the foundations of quantum mechanics; and laser theory, particularly as it applies to conceptual designs for the $\gamma$-ray laser. He has authored over 170 technical papers.

Concurrently with his research, Dr. Solem has held management positions continuously between 1971 and 1987. Some of his line management positions were: Group Leader of Thermonuclear Weapons Physics (1972-1976), Group Leader of Neutron Physics (1977-1979), Group Leader of High-Energy-Density Physics (1978), Deputy Division Leader of Physics (1978-1980), and Associate Division Leader of Theory (1980-1987). Some of his program management positions were: Program Manager of Bluebeard (1973-1976); Program Manager of Vanguard (19741975); and Program Manager of High-Energy-Density Physics (1978); Program Manager of SDI New and Innovative Concepts High-Energy-Density Physics (1978).

He has been a member of the USAF Scientific Advisory Board, the Munitions and Armament Panel, the Weapons Program Review Committee a consultant to the RAND Corporation; and participated on many studies concerning defense and science policy. 